\title{
Influence of oral supplementation with sesamin on longevity of Caenorhabditis elegans and the host defense
}

Yukie Yaguchi, Tomomi Komura, Noriko Kashima, Miho Tamura, Eriko Kage-Nakadai, Shigeru Saeki, Keiji Terao \& Yoshikazu Nishikawa

\begin{tabular}{|c|l|}
\hline Citation & European Journal of Nutrition. 53(8); 1659-1668 \\
\hline Issue Date & $2014-12$ \\
\hline Type & Journal Article \\
\hline Textversion & author \\
\hline Rights & $\begin{array}{l}\text { This is a post-peer-review, pre-copyedit version of an article published in European } \\
\text { Journal of Nutrition. The final authenticated version is available online at: } \\
\text { https://doi.org/10.1007/s00394-014-0671-6 }\end{array}$ \\
\hline DOI & $10.1007 / \mathrm{s} 00394-014-0671-6$ \\
\hline
\end{tabular}

\author{
Self-Archiving by Author(s) \\ Placed on: Osaka City University
}

YAGUCHI, Y., KOMURA, T., KASHIMA, N., TAMURA, M., KAGE-NAKADAI, E., SAEKI, S., TERAO, K., \& NISHIKAWA, Y. (2014). Influence of oral supplementation with sesamin on longevity of Caenorhabditis elegans and the host defense. European Journal of Nutrition. 53, 1659-1668. https://doi.org/10.1007/s00394-014-0671-6 
1 Influence of oral supplementation with sesamin on longevity of Caenorhabditis elegans and the host

2 defense

3

4 Yukie Yaguchi $\cdot$ Tomomi Komura $\cdot$ Noriko Kashima $\cdot$ Miho Tamura $\cdot$ Eriko Kage-Nakadai $\cdot$ Shigeru

$5 \quad$ Saeki $\cdot$ Keiji Terao $\cdot$ Yoshikazu Nishikawa

6

7

8 Y. Yaguchi $\cdot$ T. Komura $\cdot$ N. Kashima $\cdot$ M. Tamura $\cdot$ S. Saeki $\cdot$ Y. Nishikawa (®)

$9{ }^{1}$ Department of Food and Human Health Sciences, Graduate School of Human Life Science, Osaka City

10 University, 3-3-138 Sugimoto, Sumiyoshi-ku, Osaka 558-8585, Japan

11 e-mail: nisikawa@life.osaka-cu.ac.jp

12

13 E. Kage-Nakadai

14 The OCU Advanced Research Institute for Natural Science and Technology, Osaka City University,

15 3-3-138 Sugimoto, Sumiyoshi-ku, Osaka 558-8585, Japan

16

17 K. Terao

18 CycloChem Co., Ltd., KIBC 654R, 5-5-2 Minatojima Minamimachi, Chuo-ku, Kobe 650-0047, Japan 19 


\section{$1 \quad$ Abstract}

2 Purpose Nutritional control has been proposed as a potential therapy for slowing the senescence of immune

3 function and decreasing mortality. This study investigated whether sesamin could modify host defense

4 systems and extend the lifespan of the nematode Caenorhabditis elegans.

5 Methods Nematodes were fed standard food (the bacterium Escherichia coli strain OP50) supplemented

6 with various doses of sesamin/ $\gamma$-cyclodextrin inclusion compounds starting from young adulthood. The

7 mean lifespan, muscle function, lipofuscin accumulation, protein carbonyl content, and stress resistance of

8 the worms were examined. Then, $C$. elegans mutants harboring loss-of-function lesions in longevity- and

9 host defense-related signaling pathways were supplemented with sesamin to identify the genes involved in

10 the longevity effects.

11 Results Worms supplemented with sesamin displayed higher locomotion and prolongevity, and produced

12 offspring at levels similar to unsupplemented control animals. The growth curves of nematodes were

13 similar to those of controls, suggesting that sesamin did not induce prolongevity effects through dietary

14 restriction. Notably, sesamin made the worms more resistant to infection by Legionella pneumophila and

15 more resistant to oxidative stressors such as paraquat and hydrogen peroxide, and prolonged the lifespan of

16 a mev-1 mutant that produces abundant superoxide anions. However, the accumulation of protein carbonyls

17 and lipofuscin was similar in sesamin-exposed and control worms, suggesting that sesamin is unlikely to

18 work simply as an antioxidant. Sesamin supplementation failed to extend the lifespan of loss-of-function

19 mutants of daf-2, daf-16, pmk-1, and skn-1.

20 Conclusions Sesamin enhances the host defense of $C$. elegans and increases the average lifespan via 21 activation of both $s k n-1$ (encoding a component of the p38 MAPK pathway) and daf-16 (encoding a component of the IGF-1 pathway).

24 Keywords: Longevity, Nematodes, Sesamin, Aging, Host defense 


\section{Introduction}

3 Senescence of the immune system leads to increased infections, malignancy, and autoimmunity [1-3].

4 Indeed, the mortality of elderly people suffering from viral and bacterial infections is higher than that of younger people [4]. If the senescence of host defense could be slowed through immunonutritional intervention, then the mortality due to infectious diseases might be decreased. However, the difficulty of establishing a suitable animal model has limited studies on this topic. Although an increase of biomarkers related to immunological function has been observed in the elderly with improved nutrition [5], few reports have shown beneficial influences of nutrition on host defense, response to experimental stress, or lifespan [6-8].

Lignans constitute the major class of polyphenols in sesame seeds, and sesamin, sesamolin, and sesaminol are the most abundant of the lignans. Sesamin, with the highest abundance among the lignans, is particularly noteworthy since the compound has been associated with a variety of health effects such as anti-allergenic [9], anti-carcinogenic [10], anti-hypertensive [11], and hypocholesterolemic [12] effects, as well as decreases in fatty acid synthesis [13]. Although sesamin itself does not exhibit antioxidative activity in vitro, the beneficial effects of the compound are likely to be due to the antioxidative effects of sesamin derivatives generated during in vivo metabolism [14].

Caenorhabditis elegans is a small, free-living, bacteriophagous soil nematode. This organism has been extensively used as an experimental system for biological studies because of its morphological simplicity, transparent body, ease of cultivation, and amenability to genetic analysis. In addition, the short and reproducible lifespan of $C$. elegans is particularly advantageous for aging studies [15]. We previously showed that the nematode was a convenient model for investigating the senescence of host defense and the influence of food and nutrition on the system: an opportunistic human pathogen, Legionella pneumophila, was pathogenic only in old nematodes but not in young worms, and the presence of probiotic bacteria or orally supplemented antioxidant agents prolonged $C$. elegans lifespan and enhanced the host defense [16-19].

Here, we report that sesamin caused prolongevity in C. elegans. Although a positive effect of sesamin on longevity has been reported in Drosophila [20], the compound's prolongation of lifespan is more apparent in the nematode than in fruit flies. Caloric restriction is known to extend the lifespan of a wide range of organisms from yeasts to mammals [21], although the effect of caloric restriction on lifespan remains controversial in primates [22]. Based on the evaluation of worm biomarkers, including body and brood sizes [23], we assessed whether sesamin extended the lifespan via caloric restriction. While radical 
oxygen species (ROS) are recognized as key factors causing senescence because of the properties as

Formulation of sesamin compound in C. elegans.

\section{Materials and methods}

Nematode strains

\section{Bacterial strains} oxidants [24], ROS are still considered to contribute to anti-senescence by the hormesis effect [25]. Hence, we performed assays for muscle function, lipofuscin accumulation (gut autofluorescence), protein carbonyl content, and stress resistance to determine how sesamin induced the numerous beneficial effects on physiological function and longevity. Additionally, loss-of-function C. elegans mutants were grown on medium supplemented with sesamin to identify the genes involved in the longevity-promoting effects of the

C. elegans Bristol strain N2 and its derivative mutant strains were kindly provided by the Caenorhabditis Genetics Center, University of Minnesota. The mutants used in this study were KU25 pmk-1(km25), EU1 skn-1(zu67), CF1038 daf-16(mu86), CB1370 daf-2(e1370), TK22 mev-1(kn1), and TJ356, a strain harboring a daf-16::GFP transgene. Nematodes were maintained and propagated on nematode growth medium (NGM) according to standard techniques [26].

E. coli OP50 (OP) was used as the standard feed for nematode cultivation and was grown using tryptone soya agar (Nissui Pharmaceutical, Tokyo, Japan). Cultured bacteria (100 mg wet weight) were suspended in $0.5 \mathrm{ml} \mathrm{M} 9$ buffer, and $50 \mu \mathrm{l}$ of the resulting bacterial suspension then was spread on peptone-free modified NGM (mNGM) in 5.0-cm diameter plates to feed worms. Salmonella enterica subsp. enterica serovar Enteritidis strain SE1, originally isolated from a diarrheal specimen, and Legionella pneumophila serogroup 1 strain JR32 were used as pathogens and were cultured using tryptone soya agar (Nissui) and BCYE agar plates (Oxoid, Basingstoke, UK), respectively as described previously [16, 18]. 
previously [17]. Briefly, a sterile $\gamma \mathrm{CD}$ solution was prepared by filtering a nearly saturated $\gamma \mathrm{CD}$ (230 $\mathrm{mg} / \mathrm{ml})$ solution. Sterile sesamin ethanol solution $(2.5 \mathrm{mg} / \mathrm{ml})$ was prepared by filtration through an organic solvent-resistant filter (SLLG013SL, Millipore, Carrigtwohill, Ireland). One milliliter $\gamma$ CD solution was mixed with $0.1 \mathrm{ml}$ sesamin solution and stirred with a rotary mixer for $12-24 \mathrm{~h}$ at ambient temperature. The solid complex (inclusion compounds) was collected by centrifugation and weighed. Finally, the inclusion compound was suspended in M9 buffer and vortexed so that the nematodes could ingest the compounds. Approximately $1 \mathrm{mg}$ wet weight of the compound, containing $6.3 \mu \mathrm{g}$ sesamin, was spread onto each NGM plate.

Determination of C. elegans life span

Eggs were recovered from adult $C$. elegans worms after exposure to a sodium hypochlorite/sodium hydroxide solution, as previously described [27]. The egg suspension was incubated overnight at $25^{\circ} \mathrm{C}$ to allow hatching, and the resulting suspension of L1 stage worms was centrifuged at $156 \times \mathrm{g}$ for $1 \mathrm{~min}$. After removing the supernatant by aspiration, the remaining larvae were transferred onto fresh mNGM plates covered with OP and then incubated at $25{ }^{\circ} \mathrm{C}$, with the exception of the daf-2 mutant, which was maintained at $20{ }^{\circ} \mathrm{C}$. To synchronize pubescence, worms were allowed to feed on OP for 2 days until maturation, as it is well known that the reproductive system regulates aging in C. elegans [28]. Nematocidal assays were performed by adding 35 three-day-old adult worms to each mNGM plate covered with $\mathrm{OP}$ and the sesamin inclusion compound. The plates were incubated at $25^{\circ} \mathrm{C}$, and the numbers of live and dead worms were scored every $24 \mathrm{~h}$. At $25^{\circ} \mathrm{C}$, worms produce progeny that develop into adults in 3 days and it is therefore difficult to identify the original worms. To avoid over-estimating the number of living worms, the original worms were transferred daily to fresh mNGM plates for 4 days until completion of their egg-laying phase at 7 days of age. Thereafter the worms were transferred to fresh mNGM plates every second day. A worm was considered dead when it failed to respond to a gentle touch with a worm picker. Worms that crawled off the plate or died from internal hatching were considered lost and not included in the analysis [29]. Nematocidal assays are generally performed using NGM agar plates containing peptone, which allows the overlayed bacteria to proliferate. However, the composition of NGM has been reported to influence the virulence of bacteria, and the in-situ production of metabolites by bacteria growing on the medium also may be nematocidal [30]. Thus, to exclude the possibility of bacteria-induced nematocidal effects from nutrients in the medium, the nematocidal assays were performed on mNGM plates lacking peptone. Each assay was carried out in duplicate and repeated twice unless 
otherwise stated.

Mean lifespan was estimated using the formula [31]:

3

$$
\text { MLS }=\frac{1}{N} \sum_{j} \frac{x_{j}+x_{j+1}}{2} d_{j}
$$

4 where $d_{j}$ is the number of worms that died in the age interval $\left(\mathrm{x}_{\mathrm{j}}\right.$ to $\left.\mathrm{x}_{\mathrm{j}+1}\right)$, and $N$ is the total number of worms. The standard error of the estimated mean lifespan was calculated using the equation:

$$
\mathrm{SE}=\sqrt{\frac{1}{N(N-1)} \sum_{j}\left(\frac{x_{j}+x_{j+1}}{2}-\mathrm{MLS}\right)^{2} d_{j} .}
$$

Maximum lifespan was calculated as the mean lifespan of the longest-living $15 \%$ of worms in each group.

Effect of sesamin on infections

After hatching, nematodes were grown on OP for 3 days. The adult worms then were divided into two groups that were given $\gamma \mathrm{CD}$ only or were supplemented with a $\gamma \mathrm{CD}$ inclusion compound containing sesamin. Seven- or 8-day-old worms were fed Salmonella or Legionella, respectively, instead of OP, as reported previously $[16,18]$. Nematode survival was measured as described above.

Locomotory scoring of aging nematodes

The motility of worms at different ages was examined using a scoring method described in previous reports $[32,33]$. Briefly, worms were classified according to a four-point scale: class "A" worms showed spontaneous movement or vigorous locomotion in responding to prodding; class " $\mathrm{B}$ " worms did not move unless prodded or appeared to have uncoordinated movement; class " $\mathrm{C}$ " worms moved only their head and/or tail in response to prodding; and class " $D$ " worms were dead animals. A minimum of 60 worms provided with sesamin were scored.

Lipofuscin

To obtain indices of senescence, the autofluorescence of intestinal lipofuscin was analyzed from days 10 to 18 of adulthood. Briefly, randomly selected worms were washed five times in M9 buffer and then placed onto fresh mNGM plates coated with $50 \mathrm{mM}$ sodium azide to induce anesthesia. Lipofuscin 
1 autofluorescence was measured using a M165 FC fluorescence stereomicroscope (Leica Microsystems,

2 Tokyo, Japan) equipped with a DsRED filter set (excitation, 510-560 nm; emission, 590-650 nm) and a

3 Leica DFC425 C digital microscope camera. The captured data was analyzed using Leica Application Suite

4 imaging software (Version 3.7.0).

5

6 Protein carbonyl formation

7

8 Protein carbonyl groups, which served as biomarkers of oxidative stress, were measured as reported 9 previously [34].

11 Measurement of body size

12

13 Three-day-old adult worms were placed on mNGM plates covered with OP. The body sizes of live worms 14 were measured every $24 \mathrm{~h}$ until the animals reached 7 days of age. Images of adult nematodes were taken with a VCT-VBIT digital microscope (Shimadzu, Kyoto, Japan) and analyzed using ImageJ software. In this system, the area of a worm's projection was estimated automatically and used as an index of body size.

Brood size

Eggs isolated with a sodium hypochlorite/sodium hydroxide solution were allowed to develop to 3 days of age on mNGM plates coated with $\mathrm{OP}$ at $25^{\circ} \mathrm{C}$. Three hermaphrodites were selected and transferred to an mNGM plate covered with a lawn of BI. The parental animals were transferred every $24 \mathrm{~h}$ to fresh mNGM plates until the end of the reproductive period. The resulting progeny were left to develop for 3 days and the progeny number then was determined. Each assay was performed with five plates and repeated twice.

Stress resistance assays

Worms were grown from 3 to 7 days of age on mNGM plates with or without sesamin and then subjected to oxidative stress and heat shock assays. To conduct an oxidative stress assay, worms were transferred onto mNGM containing 1.0 or $2.5 \mathrm{mM}$ paraquat, or into M9 buffer with $0.1 \%$ cholesterol (5 mg ml${ }^{-1}$ in ethanol) that contained either $0.8 \mathrm{mM}$ hydrogen peroxide, $2.0 \mathrm{mM}$ hydrogen peroxide, or $7.0 \mathrm{mM}$ cupric chloride. 
tolerance, 7-day-old worms were placed in M9 buffer or onto mNGM at $35^{\circ} \mathrm{C}$ and then scored for viability.

2 Similarly the 7-day-old worms were exposed to UV irradiation at $250 \mathrm{~J} / \mathrm{m}^{2}$ or $500 \mathrm{~J} / \mathrm{m}^{2}$. The survival of

3 worms was determined by touch-provoked movement. Worms were scored as dead when the animals failed

4 to respond to mechanical stimuli with a worm picker. The assays were performed at least twice.

5

6 Statistical analysis

7 $<0.05$.

\section{Results} Fig. 2a, b).

Nematode survival was calculated by the Kaplan-Meier method, and survival differences were tested for significance using the log-rank test. Differences in protein carbonyl levels and brood size were determined using the Student $t$-test. Differences in lipofuscin levels were analyzed using the Mann-Whitney $\mathrm{U}$ test. Differences in body sizes were analyzed using the Kruskal-Wallis test. The level of significance was set at P

Prolongevity due to sesamin

The lifespan of nematodes that ingested sesamin-containing $\gamma \mathrm{CD}$ was $13.6 \%$ longer than that of control worms maintained on mNGM containing $\gamma \mathrm{CD}$ alone (Fig. 1; the data for Fig. 1 is provided in Online Resource Table 1). The effect was dose-dependent with the strongest effect seen at $6.3 \mu \mathrm{g} /$ plate; supplementation at higher or lower concentrations of sesamin resulted in weaker or no prolongevity effects.

Muscle function and accumulation of lipofuscin, as determined by gut autofluorescence, are known to correlate with age, but vary between age-matched individuals in C. elegans [23]. In the present study, locomotory ability was assayed as an indicator of muscle function, as locomotory class is predictive for the remaining lifespan of C. elegans worms after 8 days of age [32]. The ratio of worms displaying coordinated sinusoidal locomotion (class A) was apparently higher in the group supplemented with sesamin (Fig. 2).

Autofluorescence of lipofuscin, a lipid peroxidation product, in worms supplemented with sesamin was not significantly different from that in control worms (Online Resource Fig. 1). The abundance of protein carbonyls, which is a general biomarker of protein oxidation, in the extracts from worms given sesamin also was similar to that in control worms, and the values were not significantly different (Online Resource 
Body and brood sizes

3

4 To examine whether the prolongevity effects of sesamin were a result of caloric reduction, the body and brood sizes of nematodes supplemented with sesamin were compared with those of control worms fed OP. The supplementation with sesamin did not alter the growth curve of worms (Fig. 3). Although caloric restriction reportedly extends the lifespan by reducing brood size [35], supplementation with sesamin did not decrease the brood size of C. elegans (Fig. 4).

Stress resistance assays

The effects of sesamin on resistance to chemical, physical, and biological stresses were examined. Sesamin supplementation (at $6.3 \mu \mathrm{g} /$ plate) yielded a highly significant ( $\mathrm{P}<0.001$ compared to unsupplemented animals) increase in survival against $1.0 \mathrm{mM}$ paraquat, a known source of ROS (Fig. 5a, Online Resource Table 2a). Similarly, when 9-day-old or 15-day-old worms were maintained in M9 buffer containing 2.0 $\mathrm{mM}$ hydrogen peroxide, a known source of active oxygen, the sesamin (at $6.3 \mu \mathrm{g} /$ plate) -supplemented worms exhibited a highly significant $(\mathrm{P}<0.001$ compared to unsupplemented animals) increase in survival time (Figs. 5b and 5c, Online Resource Tables $2 b$ and 2c). However, the worms remained vulnerable to 7.0 $\mathrm{mM} \mathrm{CuCl}_{2}$ solution irrespective of the age and sesamin supplementation status (Online Resource Figs. 3a and 3b, Online Resource Tables 2d and 2e). In the case of physical stresses (Online Resource Figs. 4a to 4d, Online Resource Tables 3a and 3b), sesamin did not prolong the survival time of worms subjected to heat stress (8-day-old worms at $32{ }^{\circ} \mathrm{C}$ or 7-day-old worms at $35^{\circ} \mathrm{C}$ ) or to UV irradiation (9-day-old worms exposed to 250 or $500 \mathrm{~J} / \mathrm{m}^{2}$ )

Previously we found that the opportunistic pathogen Legionella can be virulent in worms older than 8 days old, while young worms fed on the pathogen grow as well as those fed on the standard food bacteria OP [18]. Thus, the opportunistic infection of C. elegans by Legionella can serve as a unique model to investigate immunosenescence. To study the effect of sesamin on response to biological stress, 7- or 8-day-old worms were fed Salmonella or Legionella, respectively, instead of OP. Oral supplementation with sesamin failed to enhance the host defense to Salmonella, a bacterial strain that exhibits pathogenicity irrespective of age of the nematodes (Online Resource Fig. 5, Online Resource Table 4a). However, when nematodes were exposed to the opportunistic pathogen Legionella, sesamin protected against death from the infection (Fig. 6, Online Resource Table 4b). 
Prolongevity effects in mutants

3

4 The PMK-1 (p38 mitogen-activated protein kinase; p38 MAPK) and DAF-2/DAF-16 insulin/IGF-1

5 signaling (IIS) pathways contribute to homeostasis, including host defense against pathogens in C. elegans.

6 To investigate whether these two pathways are involved in the prolongevity effects of sesamin, the lifespan of $C$. elegans loss-of-function mutants fed sesamin was compared with that of control worms fed OP. Sesamin failed to prolong the lifespan of strains harboring individual mutations in the pmk-1 (encoding an ortholog of mammalian p38 MAPK), skn-1 (encoding an ortholog of mammalian Nrf2), daf-2 (encoding an ortholog of Insulin/IGF-1-like receptor), or daf-16 (encoding an ortholog of mammalian FoxO) genes (Online Resource Figs. 6a to 6d, Online Resource Tables 5a to 5d). In contrast, sesamin prolonged the mean lifespan of the mev-1 mutant by $13 \%$ (Online Resource Fig. 6e, Online Resource Table 5e), an increase that was statistically significant $(\mathrm{P}<0.05)$. These results suggested the importance of both $\mathrm{p} 38$ MAPK and IIS pathways for mediating the prolongevity effect of sesamin. When a strain harboring a daf-16::GFP transgene was grown in the presence of sesamin, the DAF-16::GFP transcription factor exhibited translocation into the nuclei of foregut cells (Fig. 7a); although the translocation was not as intense as that observed in worms of the same strain exposed to heat stress in the absence of sesamin supplementation (Fig. 7b).

\section{Discussion}

Sesamin, a hydrophobic substance and precursor of the antioxidative sesaminol, clearly extended the lifespan of the nematodes in this study. The worms given sesamin were more locomotive than the controls during old age, and were more resistant to L. pneumophila, a species that is selectively pathogenic for aged worms. Further, sesamin protected worms from oxidative stresses produced by selected chemicals (paraquat and peroxide), though not for that associated with cupric chloride. Sesamin supplementation was not protective against physical stresses such as UV irradiation and heat stress, nor against pathogenic Salmonella. In the course of developing methods to let C. elegans ingest hydrophilic or hydrophobic chemicals, we previously had observed that several antioxidants (including $\mathrm{N}$-acetyl-cysteine, glutathione, tocotrienol, and astaxanthin) prolonged the lifespan of nematodes $[17,19,36]$. In mev-1 mutants, shortened lifespan is considered to be the result of an increased production of ROS [37]. Our obsservation, in the 
present work, of increased longevity in sesamin-treated mev- 1 animals is consistent with the proposed role of ROS in this mutant strain.

It is an attractive hypothesis that sesamin works as an antioxidant precursor, slowing senescence compared to control animals, and thereby resulting in prolongevity and increased resistance to the opportunistic pathogen Legionella. Oxidative stress is typically associated with protein modifications (including carbonylation of amino acid side chains), and the accumulation of lipofuscin serves as a marker of aging. However, there were no signficant differences in the levels of lipofuscin or carbonylated protein between sesamin-supplemented worms and age-matched controls. Thus, sesamin anti-oxidant activity does not appear to affect oxidants produced endogenously in the worms. Sesamin may reduce harm caused by exogenous oxidants; although it remains unclear why the supplementation failed to yield protective effects against copper chloride, a compound that is expected to induce exogenous ROS production [38, 39]. If sesamin does not play a direct role as an antioxidant, sesamin is expected to render worms indirectly resistant to the oxidative chemicals used in this study. Among these indirect roles, antioxidants can work as pro-oxidant hormetins [40], a mechanism that is not expected to decrease the levels of carbonylated protein and lipofuscin. Instead, the resultant hormesis would enhance the host defense via signal induction pathways, in the same way that low levels of ROS can cause so-called redox signaling [41, 42]. This hormetic effect would explain why higher doses of sesamin were not as effective in extending worm lifespan.

Caloric restriction is well recognized as a mechanism for the extension of longevity, and such restriction has been shown to slow senescence not only in numerous non-mammalian taxa, but also in mammals [21]. However, sesamin does not appear to cause dietary restriction in nematodes, as evidenced by the fact that growth curves were similar regardless of sesamin exposure. Furthermore, the sesamin-supplemented nematodes produced offspring in numbers similar to those seen in the control worms. This observation confirms our hypothesis that sesamin's effects are not mediated by effects on dietary restriction, given that Bishop and Guarente [5] demonstrated that the prolongevity effect of dietary restriction reflects reductions in brood sizes. Indeed, if sesamin were causing caloric restriction, the compound should extend the lifespan of a C. elegans daf-2 mutant [35], which we did not observe in the present work.

The IIS pathway has a conserved role in modulation of lifespan, and the pathway includes DAF-16, a forkhead family transcription factor that regulates genes that promote stress resistance and extend the lifespan of nematodes [43]. Since sesamin failed to prolong the lifespan of the daf-2 or daf-16 mutants, this pathway is presumably involved in the prolongevity effects of sesamin. Similarly, sesamin failed to prolong 
1 the lifespan of the C. elegans pmk-1 and skn- 1 mutants, suggesting that these genes, components of the p38

2 MAPK pathway, mediate the prolongevity effect of the compound. Thus, sesamin appears to promote the 3 induction of phase-2 detoxification enzymes in C. elegans through activation of SKN-1, an ortholog of mammalian Nrf transcription factors [44]. As SKN-1 appears to be involved in damage control and stress resistance [45], endogenous antioxidant systems upregulated via activated SKN-1 may account for the enhanced resistance to stressors and the extension of lifespan. Both the p38 MAPK and IIS pathways are evidently required for the effects of sesamin (Figure 8).

Inhibition of the TOR (target of rapamycin) is another well-known intervention method for prolongevity: dietary restriction / caloric restriction is likely to extend lifespan by inhibiting the TOR kinase. This protein, which is essential for growth but associated with aging, forms two distinct complexes, designated TORC1 and TORC2. Recently, Robida-Stubbs et al. [38] showed that TORC1 inhibition resulted in extension of lifespan in C. elegans, a process that depended on both the p38 MAPK and IIS pathways. In contrast, while TORC2 inhibition also increased life span, this process required SKN-1 but not DAF-16 [46]. We previously observed that the presence of bifidobacteria extended lifespan in $C$. elegans and suppressed the age-associated increase of the sensitivity to Salmonella or Legionella [16, 18]. Unlike sesamin, however, bifidobacteria elicit their effects mainly via the p38 MAPK pathway, and not via the IIS pathway. The lower lipofuscin and protein carbonyl levels in the worms fed bifidobacteria were taken as evidence of enhanced antioxidant systems, although the bifidobacteria-eating worms were as vulnerable to the chemical and physical stresses used in that study as the control worms. The distinction between the effects of sesamin and those of bifidobacteria might reflect differential effects on the TOR pathway. In this model, sesamin would be a mimetic of caloric restriction, which is known to decrease the activity of TORC1.

In conclusion, the present study suggests that sesamin extends the lifespan of $C$. elegans via modulation of both the p38 MAPK and IIS pathways, possibly by suppression of TORC1, although this effect is not mediated by caloric restriction per se. We note that sesamin seems to function as a hormetin rather than as an antioxidant, and that the compound enhances the host defense of nematodes against a subset of chemical oxidative stressors and opportunistic pathogens. Agents that promote particular SKN-1 or DAF-16 activities likely cause beneficial effects of TOR inhibition without interfering with critical TOR functions and inducing side effects, in contrast to rapamycin, which is known to impair immune function and reduce insulin sensitivity. The role of sesamin as a possible suppressor of TORC1 will be the subject of further studies. We additionally propose that $C$. elegans will serve as a useful model for the identification and characterization of other TOR inhibitors. 


\section{Acknowledgements}

4

5 This study was supported in part by a Grant-in-aid for Scientific Research C (No. 23617017) and a

6 Grant-in-aid for Scientific Research A (No. 23247045) from the Japan Society for the Promotion of Science.

7 The nematodes used in this study were kindly provided by the Caenorhabditis Genetics Center, which is 8 funded by the NIH National Center for Research Resources (NCRR).

9 On behalf of all authors, the corresponding author states that there is no conflict of interest. 


\section{References}

3 1. Pawelec G, Larbi A (2008) Immunity and ageing in man: Annual review 2006/2007. Exp Gerontol

2. Grubeck-Loebenstein B (1997) Changes in the aging immune system. Biologicals 25:205-208

3. Moulias R, Devillechabrolle A, Lesourd B, Proust J, Marescot MR, Doumerc S, Favre Berrone M, Congy F, Wang A (1985) Respective roles of immune and nutritional factors in the priming of the immune response in the elderly. Mech Ageing Dev 31:123-137

4. Bradley SF, Kauffman CA (1990) Aging and the response to salmonella infection. Exp Gerontol $25: 75-80$

5. Bogden JD, Louria DB (2004) Nutrition and immunity in the elderly. In: Hughes DA, Gail

6. Fulop T, Larbi A, Hirokawa K, Mocchegiani E, Lesourd B, Castle S, Wikby A, Franceschi C, Pawelec G (2007) Immunosupportive therapies in aging. Clin Intervent Aging 2:33-54

8. Effros RB, Walford RL, Weindruch R, Mitcheltree C (1991) Influences of dietary restriction on immunity to influenza in aged mice. J Gerontol 46:B142-147

10. Hirose N, Doi F, Ueki T, Akazawa K, Chijiiwa K, Sugano M, Akimoto K, Shimizu S, Yamada H

11. Matsumura Y, Kita S, Tanida Y, Taguchi Y, Morimoto S, Akimoto K, Tanaka T (1998)

12. Hirata F, Fujita K, Ishikura Y, Hosoda K, Ishikawa T, Nakamura H (1996) Hypocholesterolemic effect of sesame lignan in humans. Atherosclerosis 122:135-136

13. Ide T, Ashakumary L, Takahashi Y, Kushiro M, Fukuda N, Sugano M (2001) Sesamin, a sesame lignan, decreases fatty acid synthesis in rat liver accompanying the down-regulation of sterol 
regulatory element binding protein-1. Biochim Biophys Acta 1534:1-13

14. Yasuda K, Sakaki T (2012) How is sesamin metabolised in the human liver to show its biological effects? Expert Opin Drug Metab Toxicol 8:93-102

15. Finch CE, Ruvkun G (2001) The genetics of aging. Annu. Rev. Genomics Hum. Genet. 2:435-462

16. Ikeda T, Yasui C, Hoshino K, Arikawa K, Nishikawa Y (2007) Influence of lactic acid bacteria on longevity of Caenorhabditis elegans and host defense against Salmonella entetica serovar Enteritidis. Appl Environ Microbiol 73:6404-6409

17. Kashima N, Fujikura Y, Komura T, Fujiwara S, Sakamoto M, Terao K, Nishikawa Y (2012) Development of a method for oral administration of hydrophobic substances to Caenorhabditis elegans: pro-longevity effects of oral supplementation with lipid-soluble antioxidants. Biogerontology 13:337-344

18. Komura T, Yasui C, Miyamoto H, Nishikawa Y (2010) Caenorhabditis elegans as an alternative model host for Legionella pneumophila and the protective effects of Bifidobacterium infantis. Appl Environ Microbiol 76:4105-4108

19. Shibamura A, Ikeda T, Nishikawa Y (2009) A method for oral administration of hydrophilic substances to Caenorhabditis elegans: effects of oral supplementation with antioxidants on the nematode lifespan. Mech Ageing Dev 130:652-655

20. Zuo Y, Peng C, Liang Y, Ma KY, Chan HY, Huang Y, Chen ZY (2013) Sesamin extends the mean lifespan of fruit flies. Biogerontology 14:107-119

21. Ingram DK, Zhu M, Mamczarz J, Zou S, Lane MA, Roth GS, deCabo R (2006) Calorie restriction mimetics: an emerging research field. Aging Cell 5:97-108

22. Austad SN (2012) AGEING Mixed results for dieting monkeys. Nature 489:210-211

23. Pincus Z, Slack FJ (2010) Developmental biomarkers of aging in Caenorhabditis elegans. Dev Dyn 239:1306-1314

24. Cannizzo ES, Clement CC, Sahu R, Follo C, Santambrogio L (2011) Oxidative stress, inflamm-aging and immunosenescence. J Proteomics 74:2313-2323

25. Ristow M, Schmeisser S (2011) Extending life span by increasing oxidative stress. Free Radic Biol Med 51:327-336

26. Stiernagle T (1999) Maintenance of C. elegans. In: Hope IA (ed) C. elegans: a practical approach. Oxford University Press, New York, p 51-67

27. Sulston J, Hodgkin J (1988) Methods. In: Wood WB, researchers TcoCe (eds) The nematode Caenorhabditis elegans. Cold Spring Harbor Laboratory Press, New York, p 587-606 
128 Hsin H, Kenyon C (1999) Signals from the reproductive system regulate the lifespan of C. elegans. Nature 399:362-366

29. Libina N, Berman JR, Kenyon C (2003) Tissue-specific activities of C. elegans DAF-16 in the regulation of lifespan. Cell 115:489-502

30. Anyanful A, Dolan-Livengood JM, Lewis T, Sheth S, Dezalia MN, Sherman MA, Kalman LV, Benian GM, Kalman D (2005) Paralysis and killing of Caenorhabditis elegans by enteropathogenic Escherichia coli requires the bacterial tryptophanase gene. Mol Microbiol 57:988-1007

31. Wu D, Rea SL, Yashin AI, Johnson TE (2006) Visualizing hidden heterogeneity in isogenic populations of C. elegans. Exp Gerontol 41:261-270

32. Hosono R, Sato Y, Aizawa SI, Mitsui Y (1980) Age-dependent changes in mobility and separation of the nematode Caenorhabditis elegans. Exp Gerontol 15:285-289

33. Gruber J, Ng LF, Fong S, Wong YT, Koh SA, Chen CB, Shui G, Cheong WF, Schaffer S, Wenk MR, Halliwell B (2011) Mitochondrial changes in ageing Caenorhabditis elegans--what do we learn from superoxide dismutase knockouts? PLoS ONE 6:e19444

34. Komura T, Ikeda T, Yasui C, Saeki S, Nishikawa Y (2013) Mechanism underlying prolongevity induced by bifidobacteria in Caenorhabditis elegans. Biogerontology

35. Bishop NA, Guarente L (2007) Two neurons mediate diet-restriction-induced longevity in $C$. elegans. Nature 447:545-549.

36. Kashima N, Fujikura Y, Komura T, Terao K, Nishikawa Y (2012) Prolongevity effects of tocotrienols: trials in Caenorhabditis elegans. In: Tan B, Watson RR, Preedy VR (eds) Tocotrienols: Vitamin E beyond tocopherols, second edition. CRC Press, Boca Raton, p 279-289

37. Ishii N, Fujii M, Hartman PS, Tsuda M, Yasuda K, Senoo-Matsuda N, Yanase S, Ayusawa D, Suzuki K (1998) A mutation in succinate dehydrogenase cytochrome b causes oxidative stress and ageing in nematodes. Nature 394:694-697

38. Villanueva A, Lozano J, Morales A, Lin X, Deng X, Hengartner MO, Kolesnick RN (2001) jkk-1 and mek- 1 regulate body movement coordination and response to heavy metals through $j n k-1$ in Caenorhabditis elegans. EMBO J 20:5114-5128

39. Barsyte D, Lovejoy DA, Lithgow GJ (2001) Longevity and heavy metal resistance in daf-2 and age-1 long-lived mutants of Caenorhabditis elegans. FASEB J 15:627-634

40. Bagnati M, Perugini C, Cau C, Bordone R, Albano E, Bellomo G (1999) When and why a water-soluble antioxidant becomes pro-oxidant during copper-induced low-density lipoprotein 
oxidation: a study using uric acid. Biochem J 340 ( Pt 1):143-152

41. Dickinson BC, Chang CJ (2011) Chemistry and biology of reactive oxygen species in signaling or stress responses. Nat Chem Biol 7:504-511

42. Putker M, Madl T, Vos HR, de Ruiter H, Visscher M, van den Berg MC, Kaplan M, Korswagen HC, Boelens R, Vermeulen M, Burgering BM, Dansen TB (2013) Redox-dependent control of FOXO/DAF-16 by transportin-1. Mol Cell 49:730-742

43. Lee SS, Kennedy S, Tolonen AC, Ruvkun G (2003) DAF-16 target genes that control C. elegans life-span and metabolism. Science 300:644-647

44. Inoue H, Hisamoto N, An JH, Oliveira RP, Nishida E, Blackwell TK, Matsumoto K (2005) The $C$. elegans p38 MAPK pathway regulates nuclear localization of the transcription factor SKN-1 in oxidative stress response. Genes Dev 19:2278-2283

45. Oliveira RP, Porter Abate J, Dilks K, Landis J, Ashraf J, Murphy CT, Blackwell TK (2009) Condition-adapted stress and longevity gene regulation by Caenorhabditis elegans SKN-1/Nrf. Aging Cell 8:524-541

46. Robida-Stubbs S, Glover-Cutter K, Lamming DW, Mizunuma M, Narasimhan SD, Neumann-Haefelin E, Sabatini DM, Blackwell TK (2012) TOR signaling and rapamycin influence longevity by regulating SKN-1/Nrf and DAF-16/FoxO. Cell Metab 15:713-724 


\section{Figure legends}

Fig. 1. Survival curves of C. elegans fed OP supplemented with sesamin at different doses as compared with the lifespan of control worms fed only OP. Each plate contained $10 \mathrm{mg}$ (wet weight) of bacteria. Worms were 3 days old on the graph's nominal Day $0 . *$, **, and $* * *$ indicate $\mathrm{P}$ values of $<0.05,<0.01$, and $<0.001$, respectively, for statistically significant differences between control worms fed only OP and worms fed OP supplemented with sesamin. Mean and maximum lifespan are presented in Online Resource Table 1.

Fig. 2. Locomotory activity of $C$. elegans fed OP supplemented with sesamin. Young adult worms fed OP for 2 days after hatching were transferred to plates containing $10 \mathrm{mg}$ of OP with or without sesamin at 6.3 $\mu \mathrm{g}$ / plate. Worms were 3 days old on the nominal Day 0. Animals were classified into four classes based on their locomotion: class A, robust, coordinated sinusoidal locomotion (white bars); class B, uncoordinated and/or sluggish movement (light gray bars); class C, no forward or backward movement, but head movements or shuddering in response to prodding (dark gray bars); and class D, dead animals (black bars). The rates of each class at the indicated time point are indicated.

Fig. 3. Growth curve of worms fed OP supplemented with sesamin $(6.3 \mu \mathrm{g} /$ plate). Images of adult nematodes were recorded using a digital microscope; the area of the worm's projection was measured using image processing software and used to generate an index of body size. The body sizes of the worms fed BI at all ratios were similar to those of the control worms fed only OP.

Fig. 4. Brood size of worms fed OP supplemented with sesamin (6.3 $\mu \mathrm{g} /$ plate). Total brood size was determined from 16 animals and the mean per two worms was calculated.

Fig. 5. Influence of sesamin-supplementation on susceptibility of worms to oxidative stresses. Worms were provided with sesamin at $6.3 \mu \mathrm{g} /$ plate from 3 days of age and then incubated with (a) $1.0 \mathrm{mM}$ paraquat from 7 days of age, (b) $2 \mathrm{mM}$ hydrogen peroxide from 9 days of age, or (c) $2 \mathrm{mM}$ hydrogen peroxide from 15 days of age. ***, Statistically significant difference compared to control worms fed OP only $(\mathrm{P}<0.001)$. Mean and maximum lifespan are presented in Online Resource Tables 2a to 2c.

Fig. 6. Survival of $C$. elegans infected with pathogenic bacteria. Worms were fed OP with or without sesamin $(6.3 \mu \mathrm{g} /$ plate) from 3 days of age. The nematodes were transferred to agar plates covered with $L$. pneumophila virulent strain JR32 at 7 or 8 days of age, respectively. The survival curves were compared with that of worms not supplemented with sesamin. $* * *$ Statistical significance at $\mathrm{P}<0.001$. Mean and maximum lifespan are presented in Online Resource Table 4a.

Fig. 7. Effects of sesamin on translocation of the DAF-16::GFP transcription factor. When the daf-16::GFP 
mutant was supplemented with sesamin $(6.3 \mu \mathrm{g} /$ plate),translocation of the DAF-16::GFP transcription factor into the nucleus was observed at 8 days of age (panel a). Note that translocation into the nucleus was limited to cells around the foregut and was of lower intensity than that seen in animals subjected to heat stress $\left(37^{\circ} \mathrm{C}\right.$ for $1 \mathrm{~h}$; panel b).

Fig. 8. Pathways predicted to be involved in the prolongevity effect of sesamin. Red lines indicate signals that upregulate or activate genes, and black lines indicate signals involved in suppression. Established pathways are shown by solid lines. Dotted lines suggest hypothetical pathways that remain to be elucidated. 


\section{Online Resource Figure legends}

Online Resource Fig. 1. Lipofuscin accumulation in the intestine of nematodes. Intestinal autofluorescence from lipofuscin in age-synchronized worms on days 17, 19, and 21 of adulthood was quantified using ImageJ software to determine the lipofuscin levels. Each bar represents the mean value for an $\mathrm{n}$ of ten worms, except for the bar corresponding to day 18 ( $\mathrm{n}=6$ OP-fed worms). The bar graph depicts the percent difference of the mean value in arbitrary units relative to that of control (OP-fed) worms on day 17. There was no statistically significant difference from control worms fed OP without sesamin (6.3 $\mu \mathrm{g} /$ plate $)$ at a P value of $<0.05$. Error bars represent the SE.

Online Resource Fig. 2. Influence of sesamin on protein oxidation evaluated using an OxyBlot kit. Non-derivatized proteins were used as negative controls for each sample. (a) Worms were grown in the absence or presence of sesamin $(6.3 \mu \mathrm{g} /$ plate); proteins recovered from lysed animals were labeled with DNP solution (Oxyblot) to detect the presence of protein carbonyls and separated on SDS-PAGE gels (lanes labeled OP and sesamin, respectively). After electrophoresis, the proteins were transferred to a PVDF membrane and visualized using a chemiluminescence detection assay. The membrane was re-probed with anti-actin antibody to correct for loading variation. (b) Band densities were quantified to determine the protein carbonyl levels using ImageJ software. The bar graph depicts the percent difference of the mean value of five independent experiments in arbitrary units relative to that of control worms fed only OP; error bars represent the SE. Actin or intensity of Coomassie Brilliant Blue R-stained bands was used to compensate for loading variation.

Online Resource Fig. 3. Influence of sesamin-supplementation on susceptibility of worms to oxidative stresses. Worms were provided with sesamin at $6.3 \mu \mathrm{g} /$ plate from 3 days of age and then incubated with (a) $7.0 \mathrm{mM} \mathrm{CuCl} 2$ solution from 9 days of age, or (b) $7.0 \mathrm{mM} \mathrm{CuCl}_{2}$ solution from the 15 days of age. ***, Statistically significant difference compared to control worms fed OP only $(\mathrm{P}<0.001)$. Mean and maximum lifespan are presented in Online Resource Tables 2d to 2e.

Online Resource Fig. 4. Influence of sesamin-supplementation on susceptibility of worms to physical stresses. Worms were provided with sesamin ( $6.3 \mu \mathrm{g} /$ plate) from 3 days of age and then (a) incubated at $32^{\circ} \mathrm{C}$ for $24 \mathrm{~h}$ at 8 days of age, (b) at $35^{\circ} \mathrm{C}$ for 8 to 10 hours at 7 days of age, (c) exposed to UV irradiation at $250 \mathrm{~J} / \mathrm{m}^{2}$, or (d) at $500 \mathrm{~J} / \mathrm{m}^{2}$ at 7 days of age. NS, not significant $(\mathrm{P}>0.05)$. Mean and maximum lifespan are presented in Online Resource Tables 3a and 3b.

Online Resource Fig. 5. Survival of C. elegans infected with pathogenic bacteria. Worms were fed OP 
with or without sesamin $(6.3 \mu \mathrm{g} /$ plate) from 3 days of age. The nematodes were transferred to agar plates covered with Salmonella Enteritidis at 7 days of age, respectively. The survival curves were compared with that of worms not supplemented with sesamin. Mean and maximum lifespan are presented in Online Resource Table 4b.

Online Resource Fig. 6. Effects of sesamin on the lifespan of C. elegans mutants. Survival curves of (a) pmk-1(km25), (b) skn-1(zu67), (c) daf-2(e1370), (d) daf-16(mu86), and (e) mev-1(kn1) mutant hermaphrodites fed OP with or without sesamin $(6.3 \mu \mathrm{g} /$ plate) from 3 days of age (nominal Day 0 in the figure). *Statistical significance at $\mathrm{P}<0.05$. Mean and maximum lifespan are presented in Online Resource Tables 5a to $5 \mathrm{e}$. 


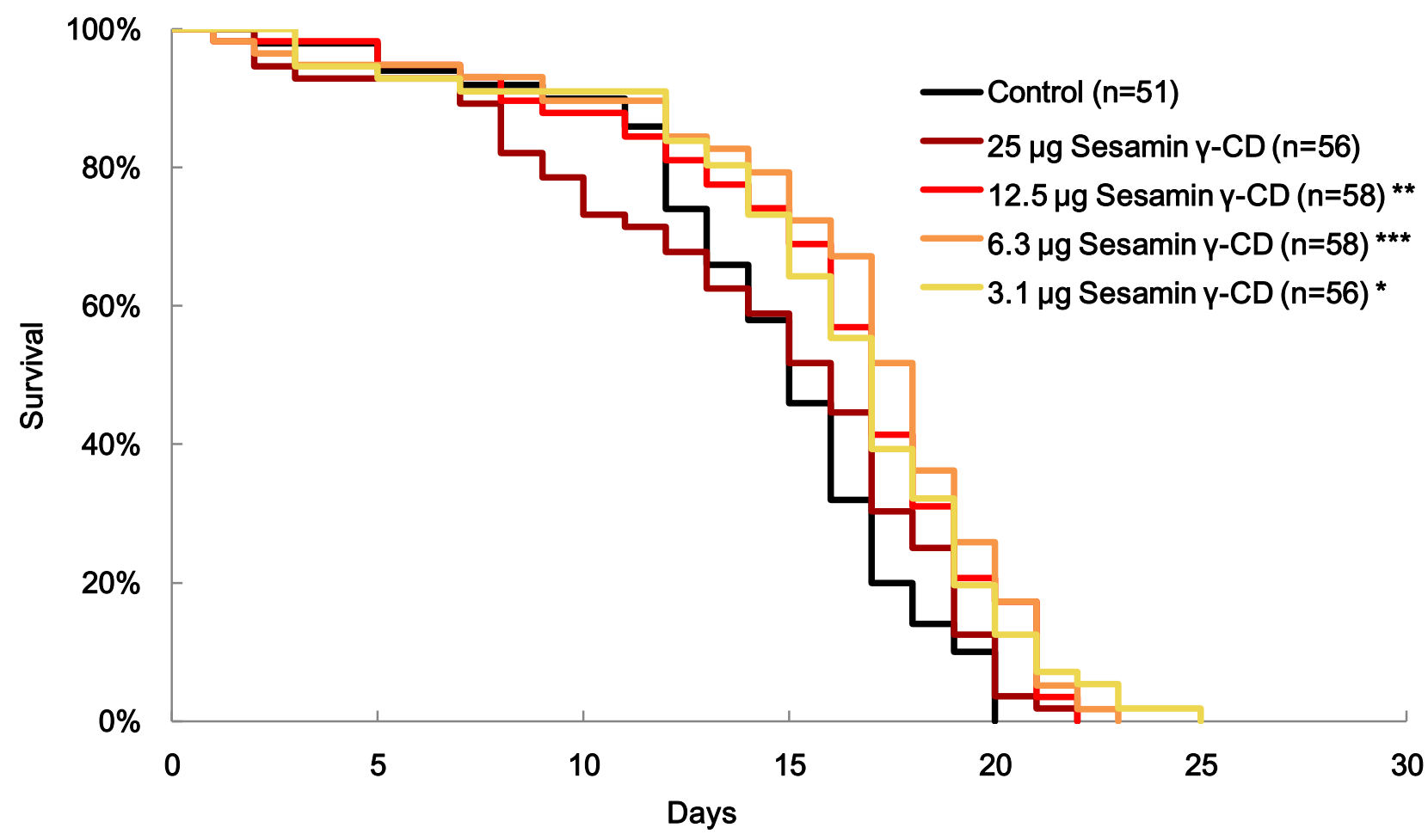

Fig. 1 


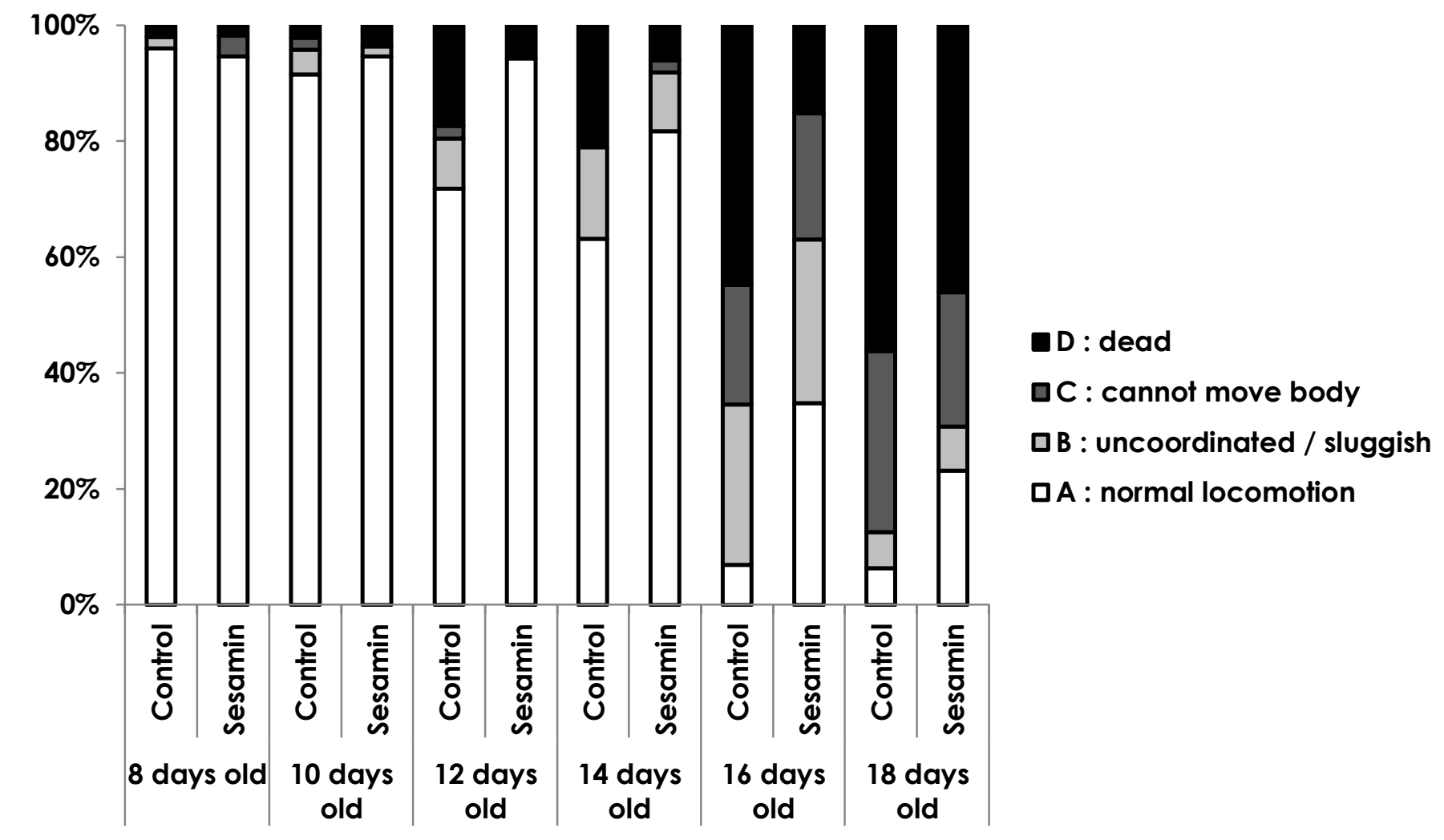

Fig. 2 


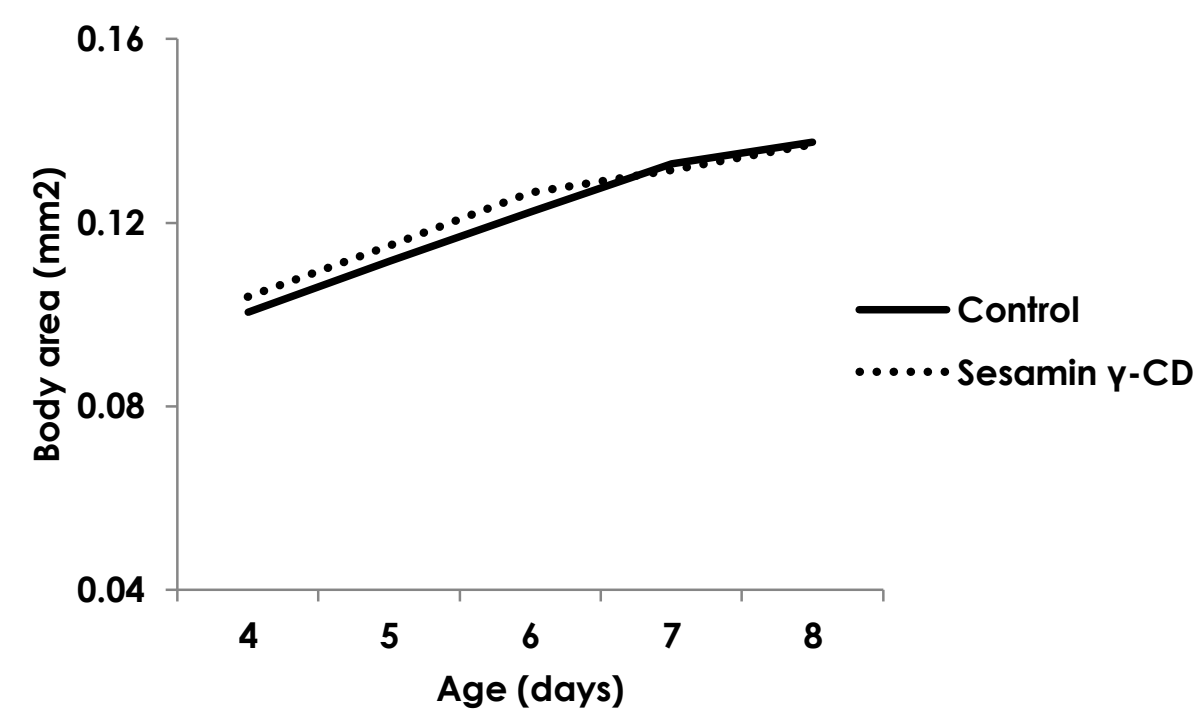

Fig. 3 


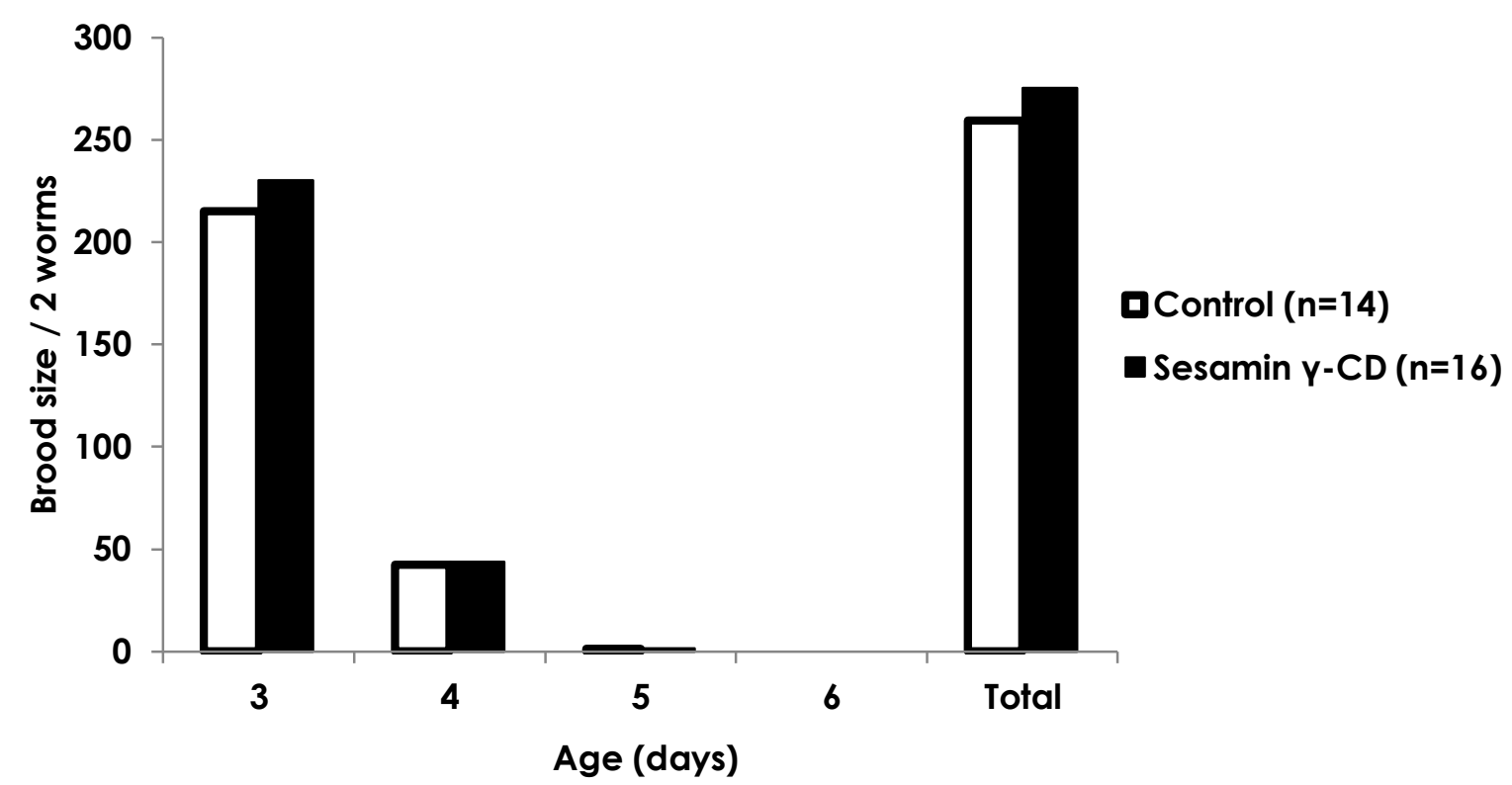

Fig. 4 


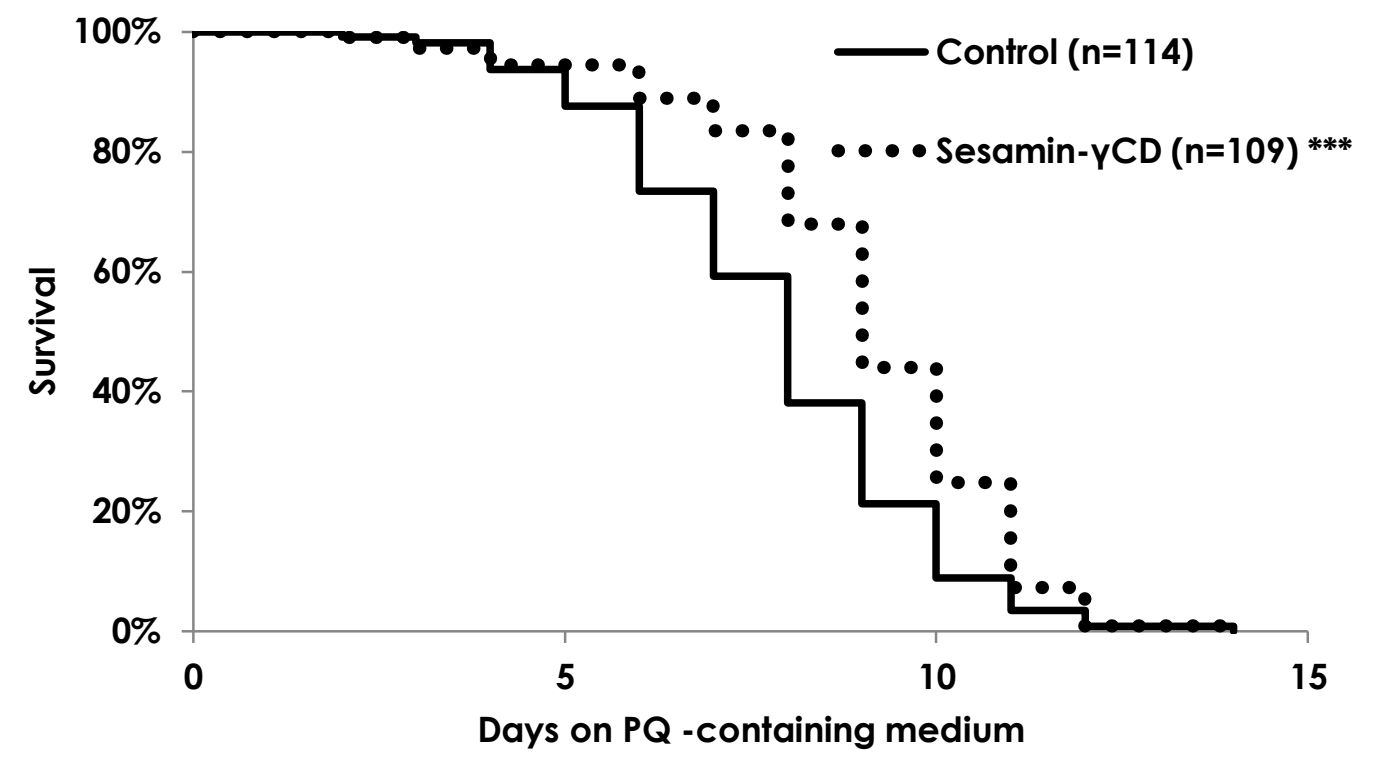

Fig. 5a 


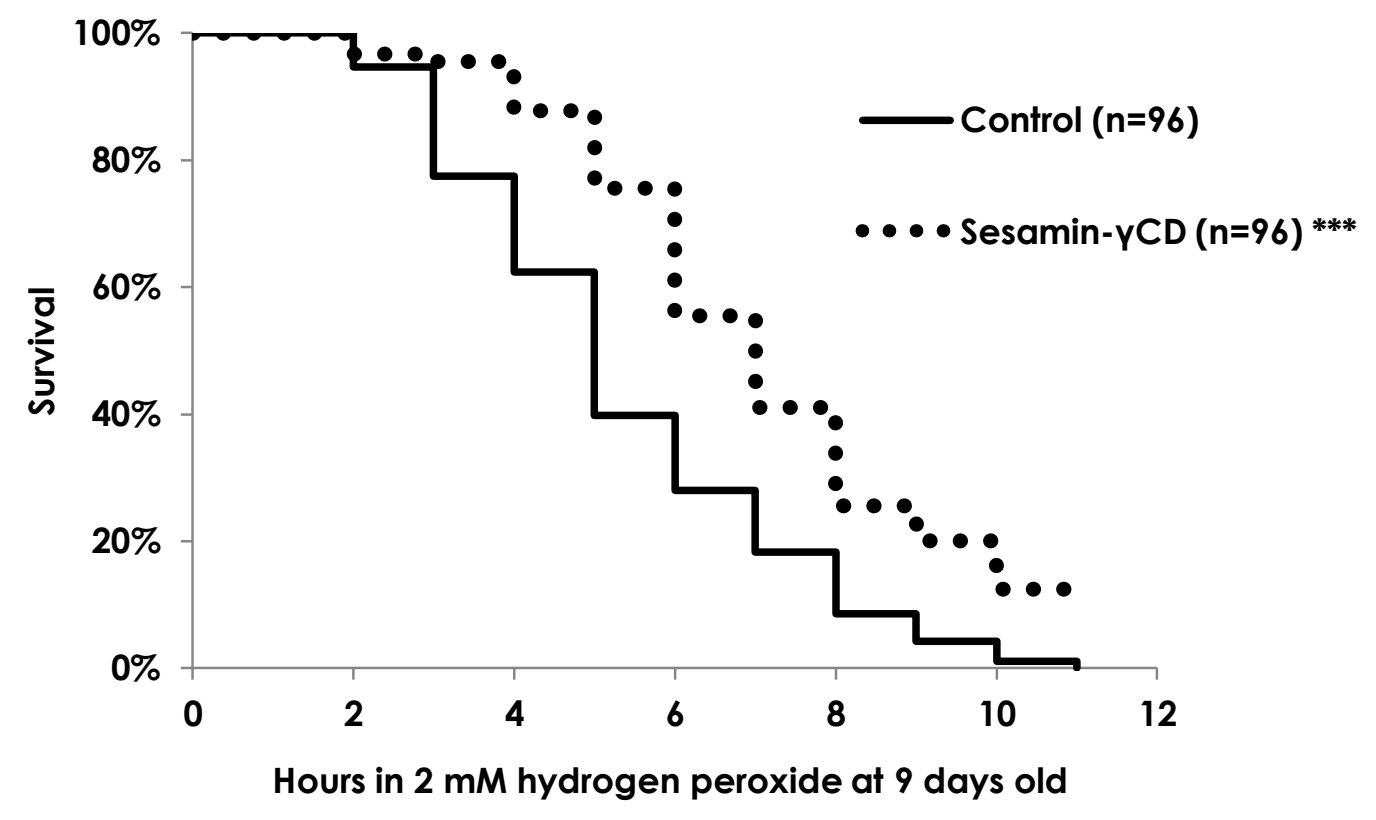

Fig. $5 b$ 


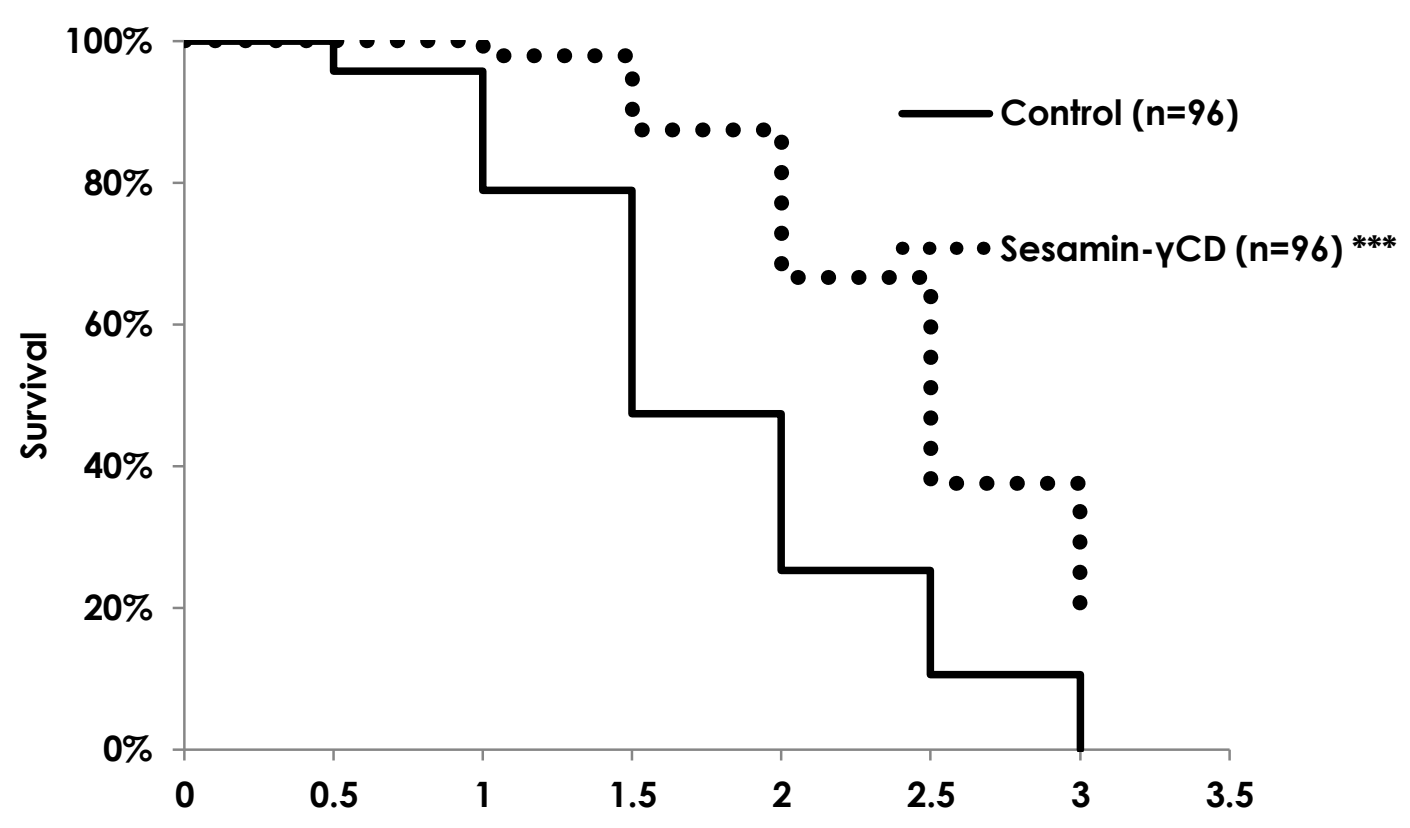

Hours in $2 \mathrm{mM}$ hydrogen peroxide at 15 days old

Fig. 5c 


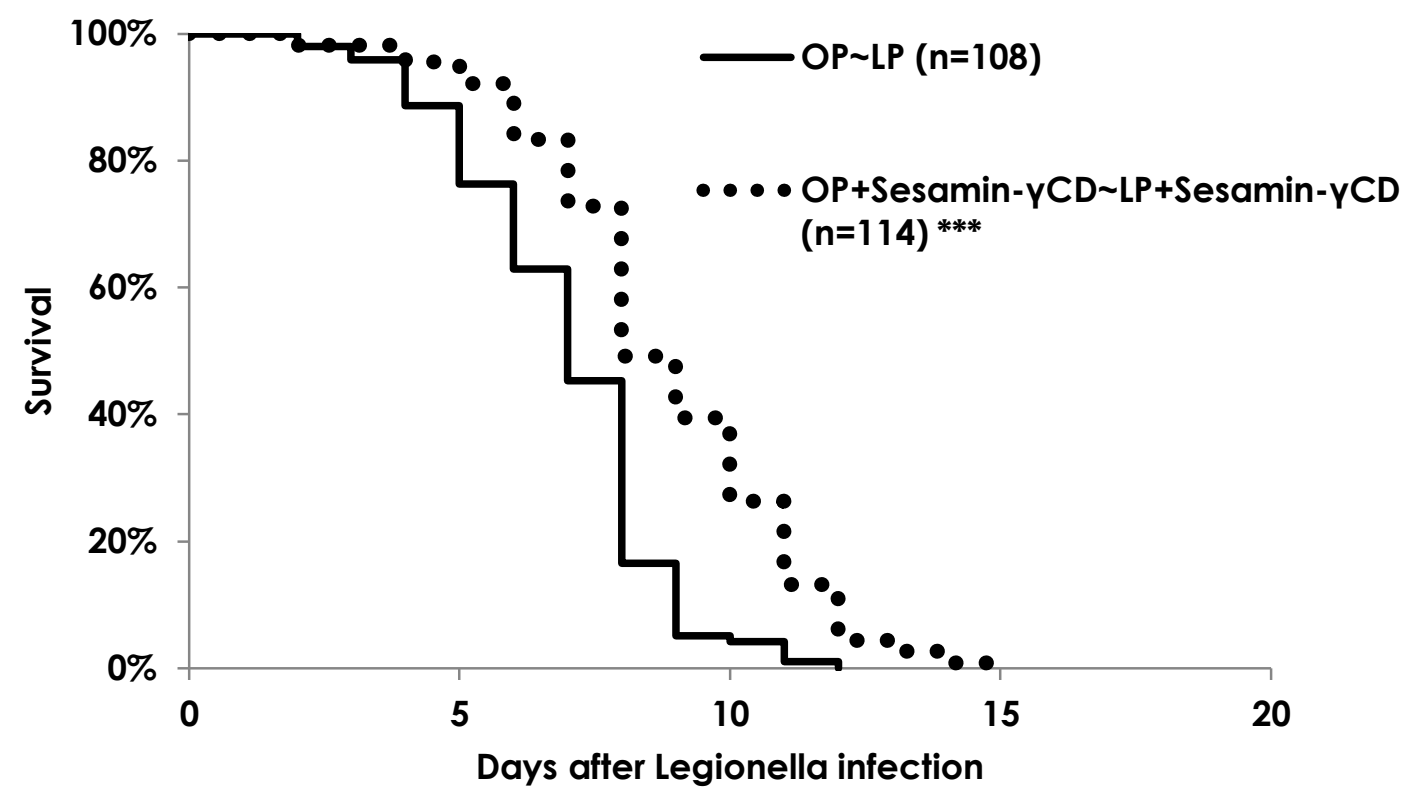

Fig. 6 

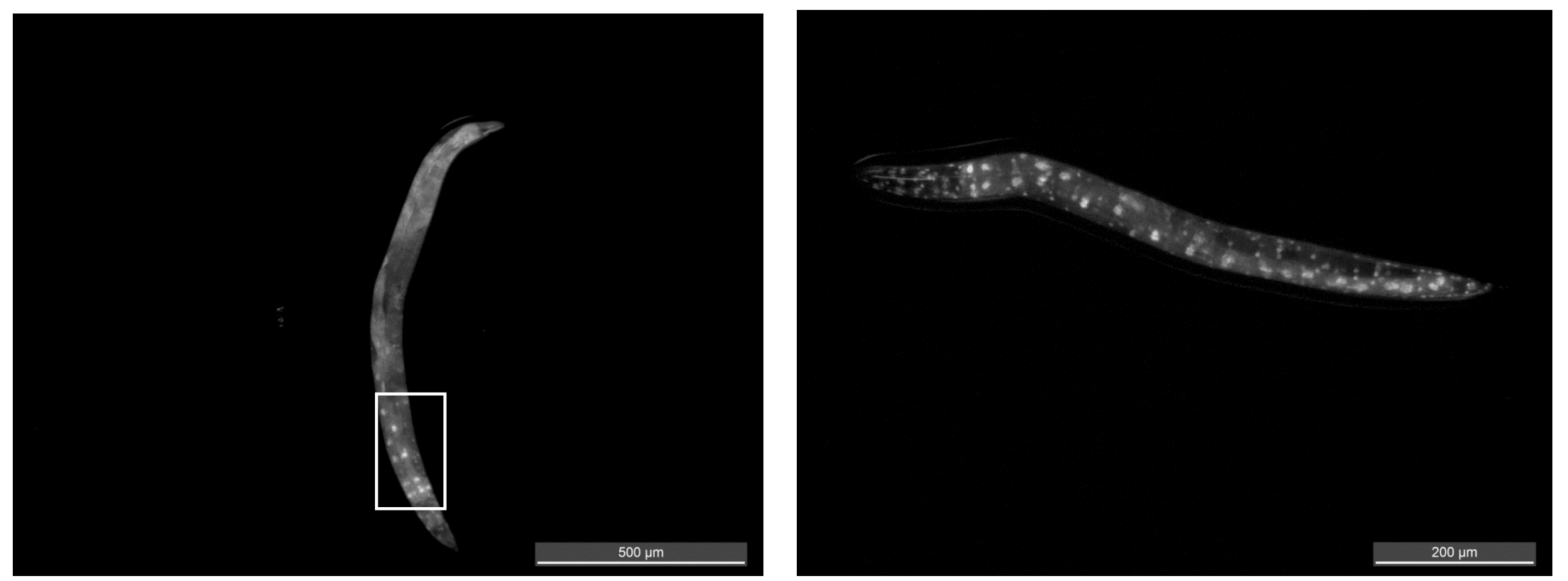

Fig. $7 \mathrm{a}$

Fig. 7b

- 9 - 


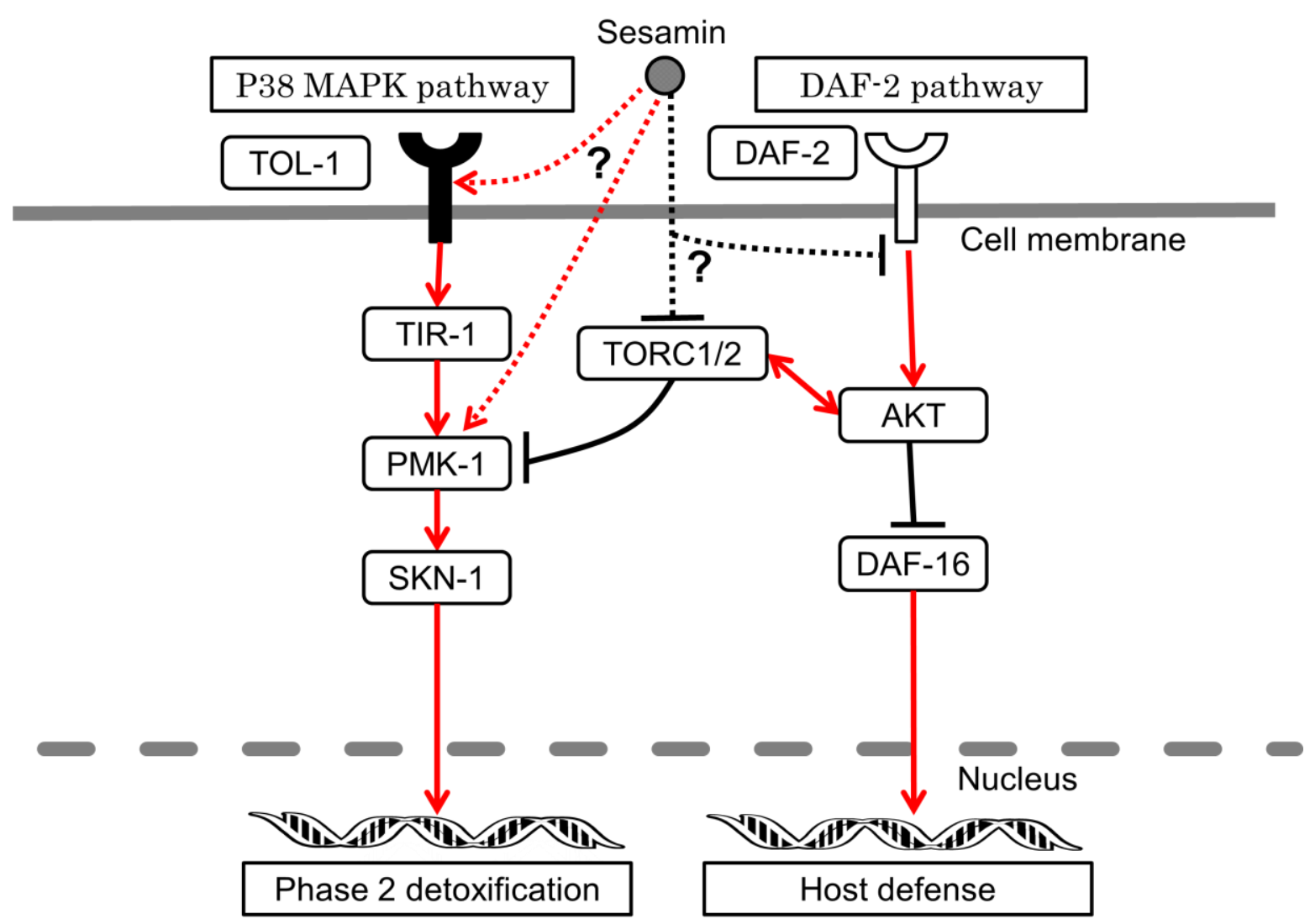

Fig. 8 
Online Resource Table 1 for Fig. 1.

\begin{tabular}{|c|c|c|c|c|c|}
\hline $\begin{array}{l}\text { Dose of } \\
\text { sesamin }\end{array}$ & $\begin{array}{c}\text { No. of } \\
\text { experiments }^{\mathrm{a}}\end{array}$ & Mean life span & $\begin{array}{c}\text { Maximum life } \\
\text { span }\end{array}$ & $\begin{array}{c}\text { No. of } \\
\text { nematodes } \\
\text { (No. of lost } \\
\text { worms) }^{b}\end{array}$ & Log-rank test \\
\hline Control & & $17.56 \pm 0.66$ & $23.00 \pm 0.27$ & $51(9)$ & \\
\hline $25 \mu \mathrm{g}$ & & $17.70 \pm 0.70$ & $23.75 \pm 0.31$ & $56(4)$ & 0.45307 \\
\hline $12.5 \mu \mathrm{g}$ & 1 & $19.48 \pm 0.61$ & $24.75 \pm 0.16$ & $58(2)$ & 0.00804 \\
\hline $6.3 \mu \mathrm{g}$ & & $20.03 \pm 0.63$ & $25.00 \pm 0.20$ & $58(2)$ & 0.00089 \\
\hline $3.1 \mu \mathrm{g}$ & & $19.41 \pm 0.62$ & $24.86 \pm 0.39$ & $56(4)$ & 0.01636 \\
\hline
\end{tabular}

${ }^{\text {a }}$ Number of independent trials. Each trial was performed using two parallel plates.

${ }^{\mathrm{b}}$ Worms that crawled off the plate or died from internal hatching were_considered lost and not included in the analysis. 
Online Resource Table 2a for Fig. 5a.

\begin{tabular}{|c|c|c|c|c|c|}
\hline Group & $\begin{array}{c}\text { No. of } \\
\text { experiments }^{\text {a }}\end{array}$ & $\begin{array}{l}\text { Mean survival } \\
\text { time } \\
\pm \text { SE (days old) }\end{array}$ & $\begin{array}{l}\text { Maximum } \\
\text { survival time } \\
\pm \text { SE (days old) }\end{array}$ & $\begin{array}{l}\text { No. of } \\
\text { nematodes } \\
\text { (No. of lost } \\
\text { worms) }^{\mathrm{b}}\end{array}$ & Log-rank test \\
\hline $\begin{array}{l}\text { Control } \\
\text { Sesamin }\end{array}$ & 2 & $\begin{array}{l}15.30 \pm 0.20 \\
16.54 \pm 0.20\end{array}$ & $\begin{array}{l}18.57 \pm 0.28 \\
19.17 \pm 0.21\end{array}$ & $\begin{array}{l}114(3) \\
109(3)\end{array}$ & 0.0009 \\
\hline
\end{tabular}

${ }^{a}$ Number of independent trials. Each trial was performed using two parallel plates.

${ }^{\mathrm{b}}$ Worms that crawled off the plate or died from internal hatching were_considered lost and not included in the analysis. 
Online Resource Table $2 b$ for Fig. $5 b$.

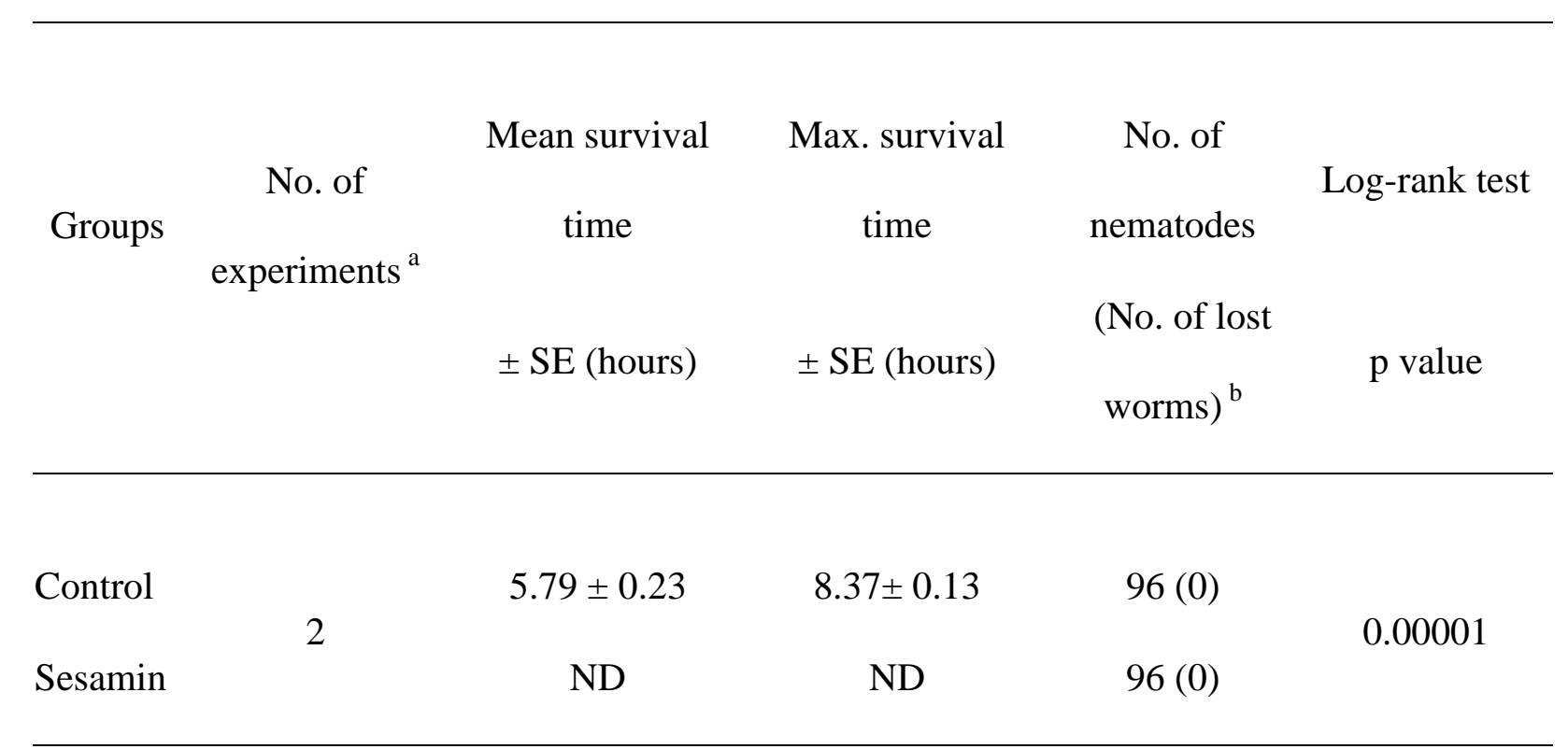

${ }^{\mathrm{a}}$ Number of independent trials. Each trial was performed using two parallel plates.

${ }^{\mathrm{b}}$ Worms that crawled off the plate or died from internal hatching were_considered lost and not included in the analysis. 
Online Resource Table 2c for Fig. 5c.

\begin{tabular}{|c|c|c|c|c|c|}
\hline \multirow[t]{2}{*}{ Groups } & \multirow{2}{*}{$\begin{array}{c}\text { No. of } \\
\text { experiments }\end{array}$} & $\begin{array}{c}\text { Mean survival } \\
\text { time }\end{array}$ & $\begin{array}{c}\text { Max. survival } \\
\text { time }\end{array}$ & $\begin{array}{c}\text { No. of } \\
\text { nematodes }\end{array}$ & \multirow{2}{*}{$\begin{array}{l}\text { Log-rank test } \\
\text { p value }\end{array}$} \\
\hline & & $\pm \mathrm{SE}$ (hours) & $\pm \mathrm{SE}$ (hours) & worms) ${ }^{b}$ & \\
\hline Control & C & $2.53 \pm 0.07$ & $3.5 \pm 0.11$ & $96(0)$ & \multirow{2}{*}{0.00000} \\
\hline Sesamin & & ND & ND & $96(0)$ & \\
\hline
\end{tabular}

${ }^{a}$ Number of independent trials. Each trial was performed using two parallel plates.

${ }^{\mathrm{b}}$ Worms that crawled off the plate or died from internal hatching were_considered lost and not included in the analysis. 
Online Resource Table 2d for Supplementary Fig. 3a.

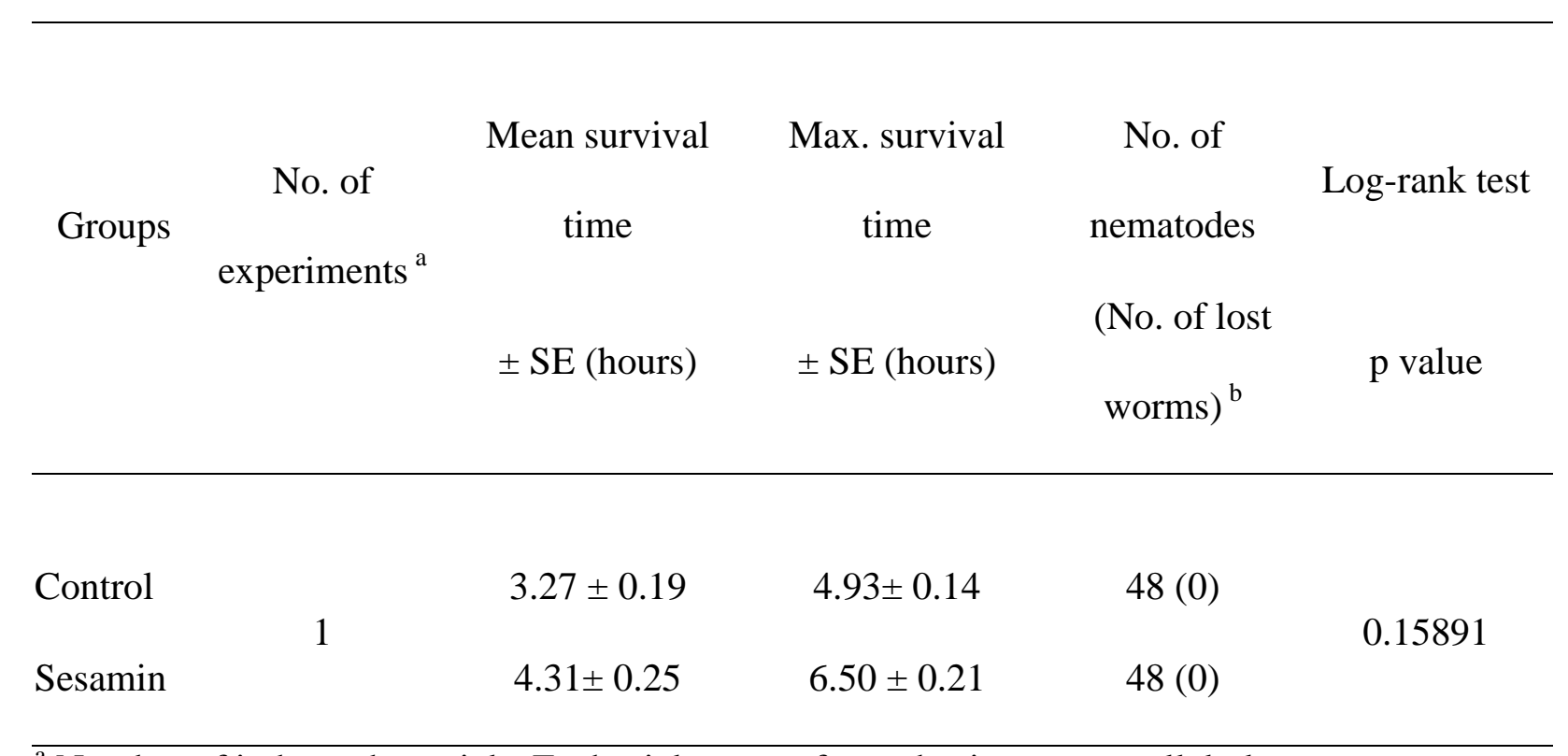

${ }^{a}$ Number of independent trials. Each trial was performed using two parallel plates.

${ }^{\mathrm{b}}$ Worms that crawled off the plate or died from internal hatching were_considered lost and not included in the analysis. 
Online Resource Table 2e for Supplementary Fig. 3b.

\begin{tabular}{|c|c|c|c|c|c|}
\hline Groups & $\begin{array}{c}\text { No. of } \\
\text { experiments }^{\text {a }}\end{array}$ & $\begin{array}{l}\text { Mean survival } \\
\text { time } \\
\pm \mathrm{SE} \text { (hours) }\end{array}$ & $\begin{array}{l}\text { Max. survival } \\
\text { time } \\
\pm \text { SE (hours) }\end{array}$ & $\begin{array}{l}\text { No. of } \\
\text { nematodes } \\
\text { (No. of lost } \\
\text { worms) }^{\mathrm{b}}\end{array}$ & $\begin{array}{c}\text { Log-rank test } \\
\text { p value }\end{array}$ \\
\hline $\begin{array}{l}\text { Control } \\
\text { Sesamin }\end{array}$ & 1 & $\begin{array}{l}1.69 \pm 0.07 \\
1.92 \pm 0.09\end{array}$ & $\begin{array}{l}2.14 \pm 0.19 \\
2.79 \pm 0.13\end{array}$ & $\begin{array}{l}48(0) \\
48(0)\end{array}$ & 0.35136 \\
\hline
\end{tabular}

${ }^{a}$ Number of independent trials. Each trial was performed using two parallel plates.

${ }^{\mathrm{b}}$ Worms that crawled off the plate or died from internal hatching were_considered lost and not included in the analysis. 
Online Resource Table 3a for Supplementary Fig. 4c.

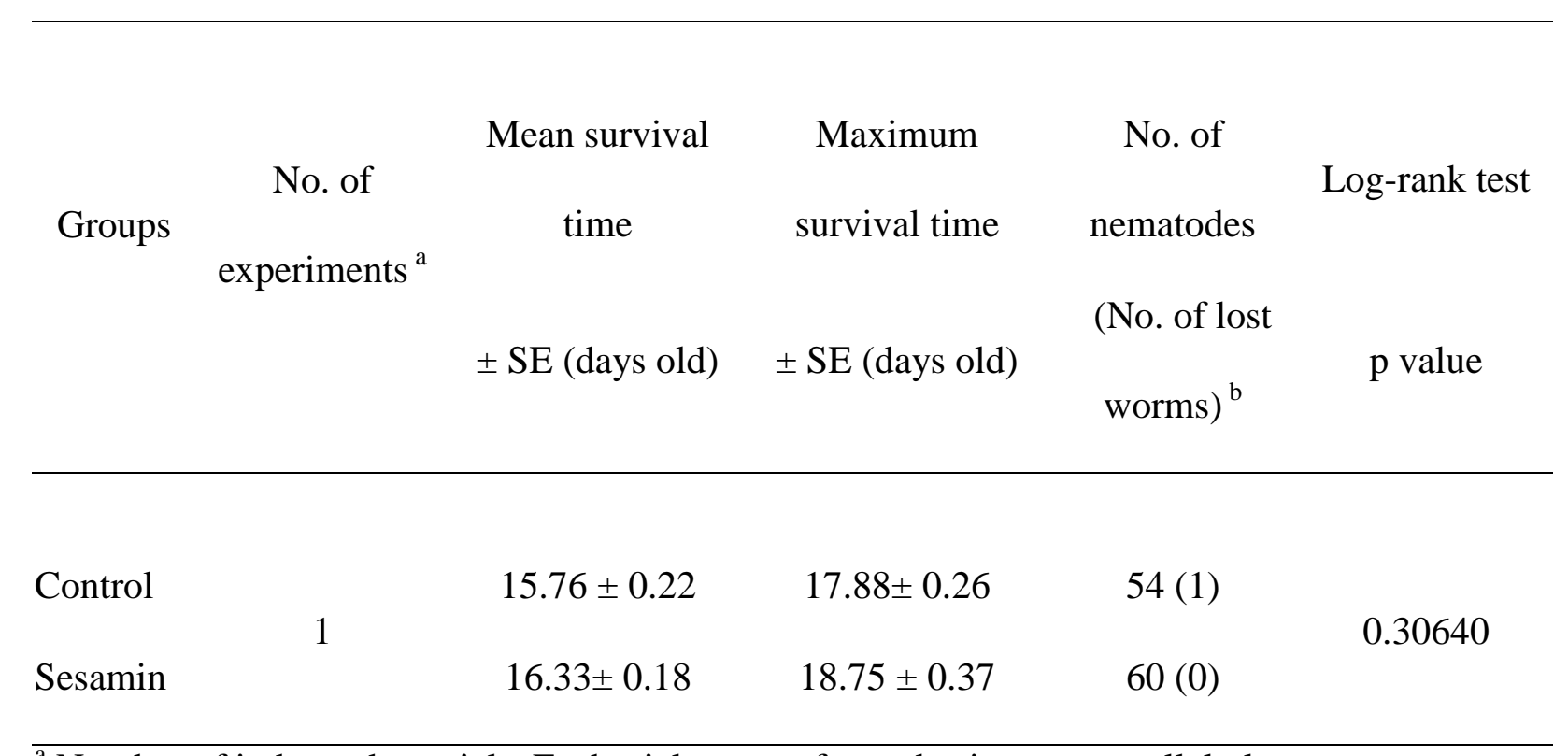

${ }^{a}$ Number of independent trials. Each trial was performed using two parallel plates.

${ }^{\mathrm{b}}$ Worms that crawled off the plate or died from internal hatching were_considered lost and not included in the analysis. 
Online Resource Table 3b for Supplementary Fig. 4d.

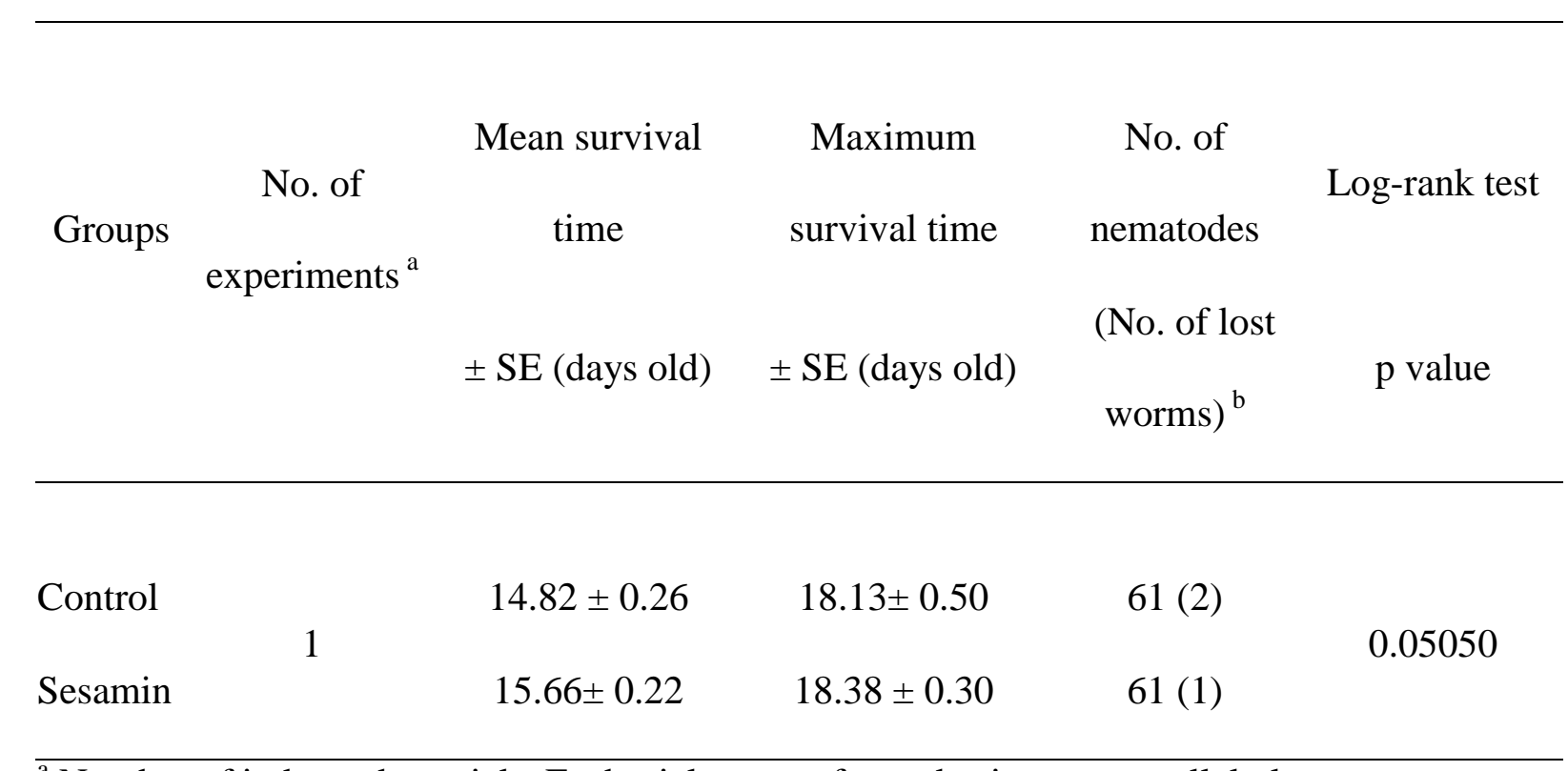

${ }^{a}$ Number of independent trials. Each trial was performed using two parallel plates.

${ }^{\mathrm{b}}$ Worms that crawled off the plate or died from internal hatching were_considered lost and not included in the analysis. 
Online Resource Table 4a for Supplementary Fig. 5.

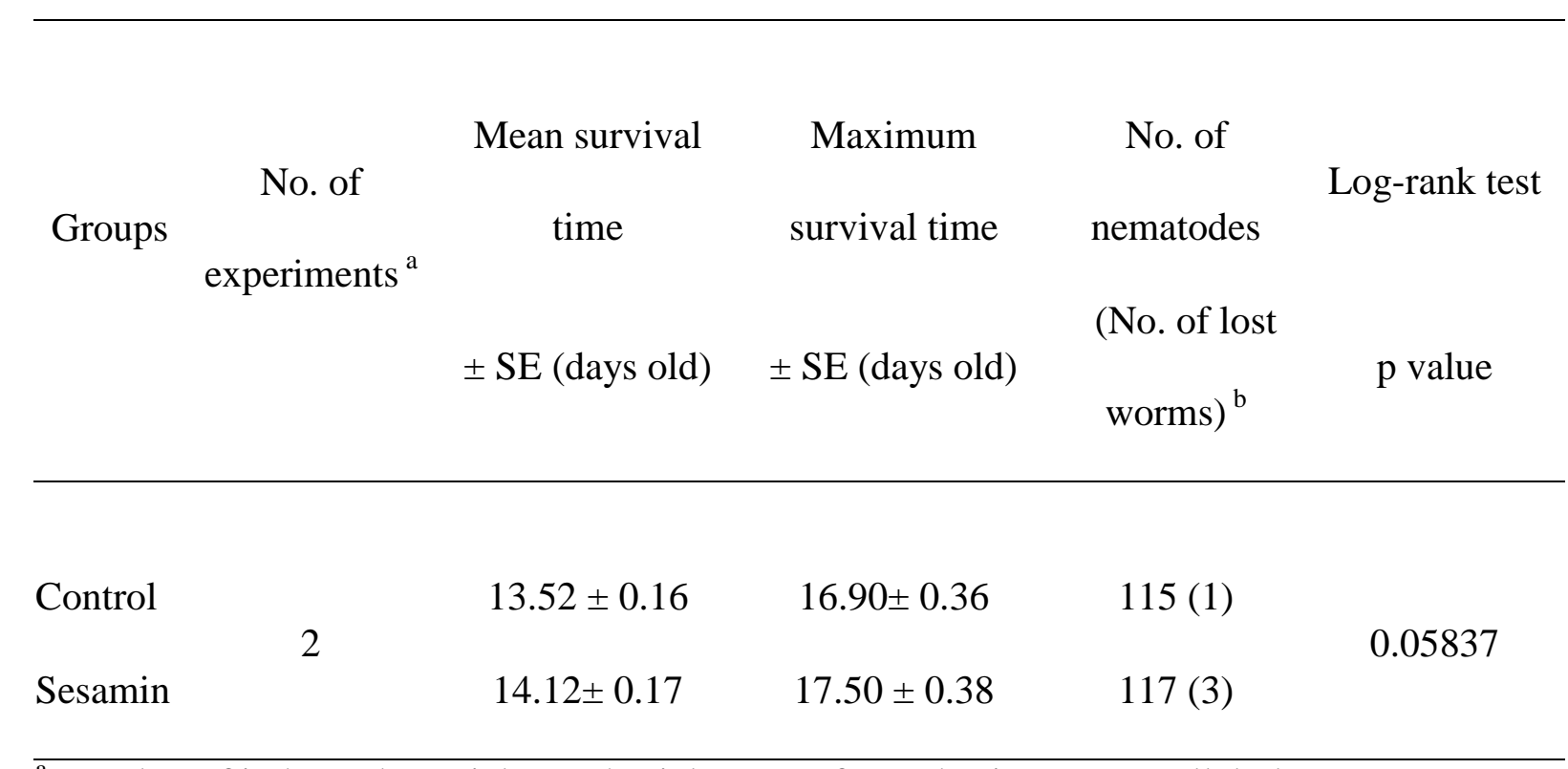

${ }^{a}$ Number of independent trials. Each trial was performed using two parallel plates.

${ }^{\mathrm{b}}$ Worms that crawled off the plate or died from internal hatching were_considered lost and not included in the analysis. 
Online Resource Table 4b for Fig. 6.

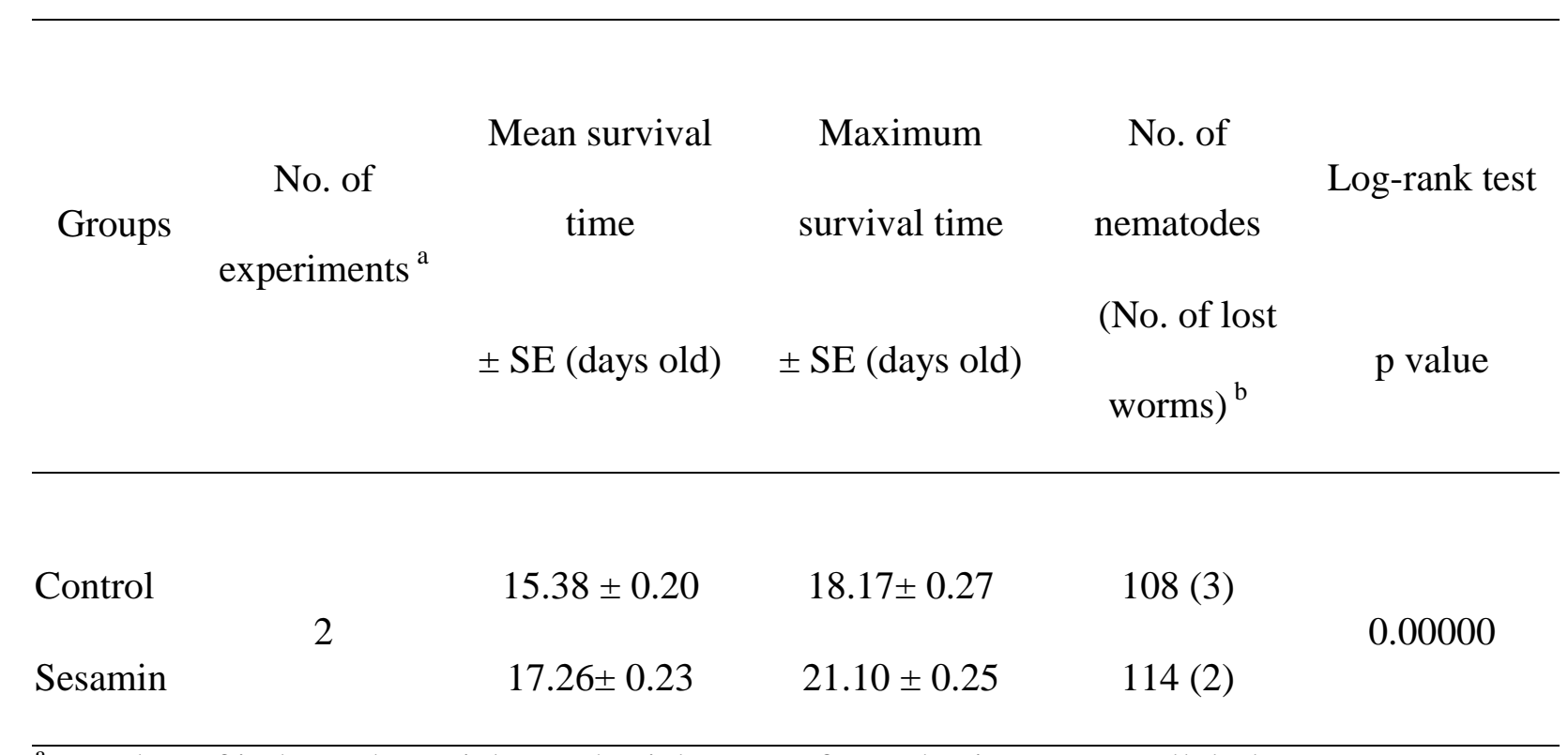

${ }^{a}$ Number of independent trials. Each trial was performed using two parallel plates.

${ }^{\mathrm{b}}$ Worms that crawled off the plate or died from internal hatching were_considered lost and not included in the analysis. 
Online Resource Table 5a for Supplementary Fig. 6a.

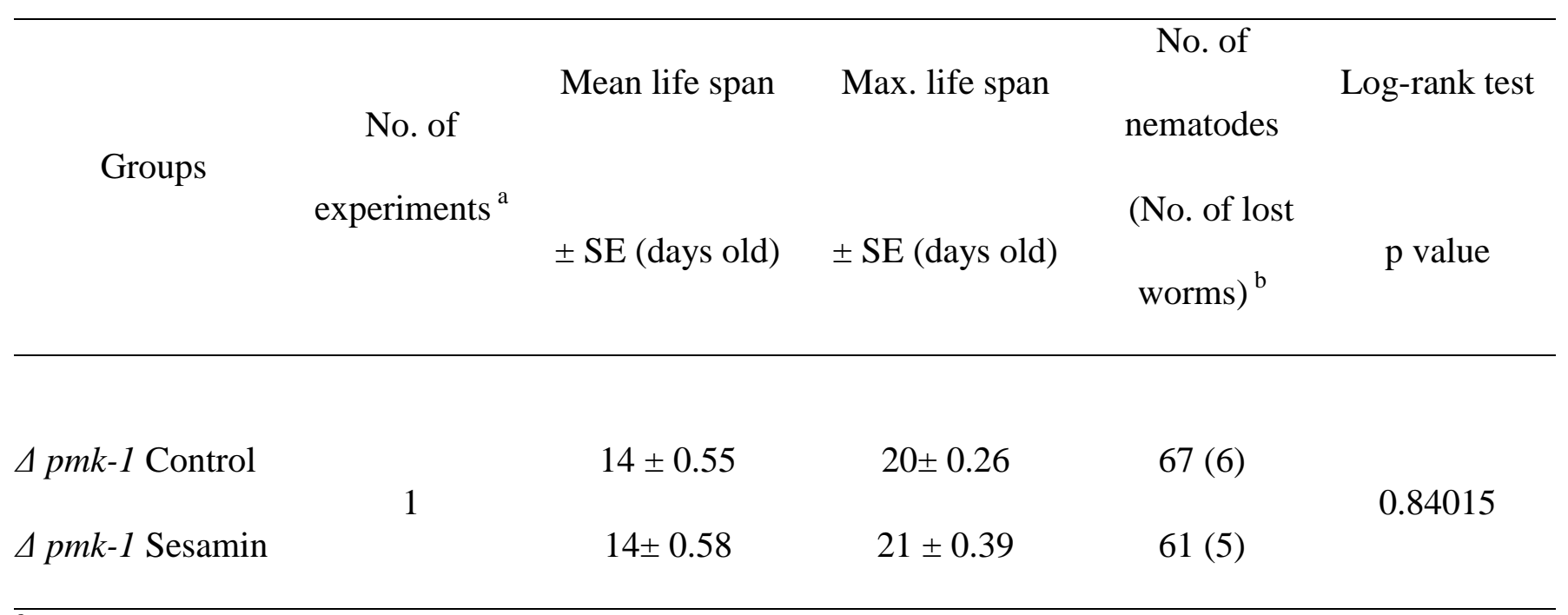

${ }^{\mathrm{a}}$ Number of independent trials. Each trial was performed using two parallel plates.

${ }^{\mathrm{b}}$ Worms that crawled off the plate or died from internal hatching were_considered lost and not included in the analysis. 
Online Resource Table 5b for Supplementary Fig. 6b.

\begin{tabular}{|c|c|c|c|c|c|}
\hline Groups & $\begin{array}{c}\text { No. of } \\
\text { experiments }^{\text {a }}\end{array}$ & $\begin{array}{l}\text { Mean life span } \\
\pm \text { SE (days old) }\end{array}$ & $\begin{array}{l}\text { Max. life span } \\
\pm \text { SE (days old) }\end{array}$ & $\begin{array}{l}\text { No. of } \\
\text { nematodes } \\
\text { (No. of lost } \\
\text { worms) }^{\mathrm{b}}\end{array}$ & $\begin{array}{l}\text { Log-rank test } \\
\text { p value }\end{array}$ \\
\hline $\begin{array}{l}\Delta s k n-1 \text { Control } \\
\Delta \text { skn-l Sesamin }\end{array}$ & 1 & $\begin{array}{l}16 \pm 0.54 \\
17 \pm 0.50\end{array}$ & $\begin{array}{l}22 \pm 0.16 \\
23 \pm 0.26\end{array}$ & $\begin{array}{l}67(1) \\
62(5)\end{array}$ & 0.29481 \\
\hline
\end{tabular}

${ }^{\text {a }}$ Number of independent trials. Each trial was performed using two parallel plates.

${ }^{\mathrm{b}}$ Worms that crawled off the plate or died from internal hatching were considered lost and not included in the analysis. 
Online Resource Table 5c for Supplementary Fig. 6c.

\begin{tabular}{|c|c|c|c|c|c|}
\hline Groups & $\begin{array}{c}\text { No. of } \\
\text { experiments }^{\text {a }}\end{array}$ & $\begin{array}{l}\text { Mean life span } \\
\pm \text { SE (days old) }\end{array}$ & $\begin{array}{l}\text { Max. life span } \\
\pm \text { SE (days old) }\end{array}$ & $\begin{array}{c}\text { No. of } \\
\text { nematodes } \\
\text { (No. of lost } \\
\text { worms) }^{\mathrm{b}}\end{array}$ & $\begin{array}{l}\text { Log-rank test } \\
\text { p value }\end{array}$ \\
\hline $\begin{array}{l}\Delta d a f-2 \text { Control } \\
\Delta d a f-2 \text { Sesamin }\end{array}$ & 1 & $\begin{array}{l}28 \pm 2.90 \\
29 \pm 3.12\end{array}$ & $\begin{array}{l}38 \pm 0.56 \\
39 \pm 0.12\end{array}$ & $\begin{array}{l}64(1) \\
68(3)\end{array}$ & 0.18890 \\
\hline
\end{tabular}

${ }^{a}$ Number of independent trials. Each trial was performed using two parallel plates.

${ }^{\mathrm{b}}$ Worms that crawled off the plate or died from internal hatching were considered lost and not included in the analysis. 
Online Resource Table 5d for Supplementary Fig. 6d.

\begin{tabular}{|c|c|c|c|c|c|}
\hline & No. of & Mean life span & Max. life span & $\begin{array}{c}\text { No. of } \\
\text { nematodes }\end{array}$ & Log-rank test \\
\hline Groups & experiments $^{\text {a }}$ & $\pm \mathrm{SE}$ (days old) & $\pm \mathrm{SE}$ (days old) & 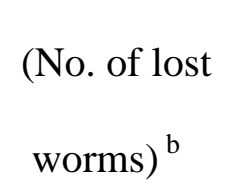 & $\mathrm{p}$ value \\
\hline $\begin{array}{l}\Delta d a f-16 \text { Control } \\
\Delta d a f-16 \text { Sesamin }\end{array}$ & 1 & $\begin{array}{l}20 \pm 0.43 \\
19 \pm 0.52\end{array}$ & $\begin{array}{l}24 \pm 0.35 \\
24 \pm 0.25\end{array}$ & $\begin{array}{l}53(6) \\
57(7)\end{array}$ & 0.41851 \\
\hline
\end{tabular}

${ }^{a}$ Number of independent trials. Each trial was performed using two parallel plates.

${ }^{\mathrm{b}}$ Worms that crawled off the plate or died from internal hatching were_considered lost and not included in the analysis. 
Online Resource Table 5e for Supplementary Fig. 6e.

\begin{tabular}{|c|c|c|c|c|c|}
\hline \multirow{2}{*}{ Groups } & No. of & Mean life span & Max. life span & $\begin{array}{c}\text { No. of } \\
\text { nematodes }\end{array}$ & Log-rank test \\
\hline & experiments $^{\mathrm{a}}$ & $\pm \mathrm{SE}$ (days old) & $\pm \mathrm{SE}($ days old $)$ & 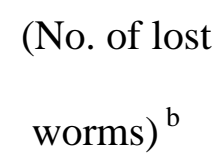 & $\mathrm{p}$ value \\
\hline$\Delta m e v-1$ Control & 2 & $14 \pm 0.52$ & $23 \pm 0.30$ & $139(10)$ & 00281 \\
\hline$\Delta m e v-1$ Sesamin & & $16 \pm 0.53$ & $24 \pm 0.34$ & $118(23)$ & \\
\hline
\end{tabular}

${ }^{\mathrm{a}}$ Number of independent trials. Each trial was performed using two parallel plates.

${ }^{\mathrm{b}}$ Worms that crawled off the plate or died from internal hatching were_considered lost and not included in the analysis. 


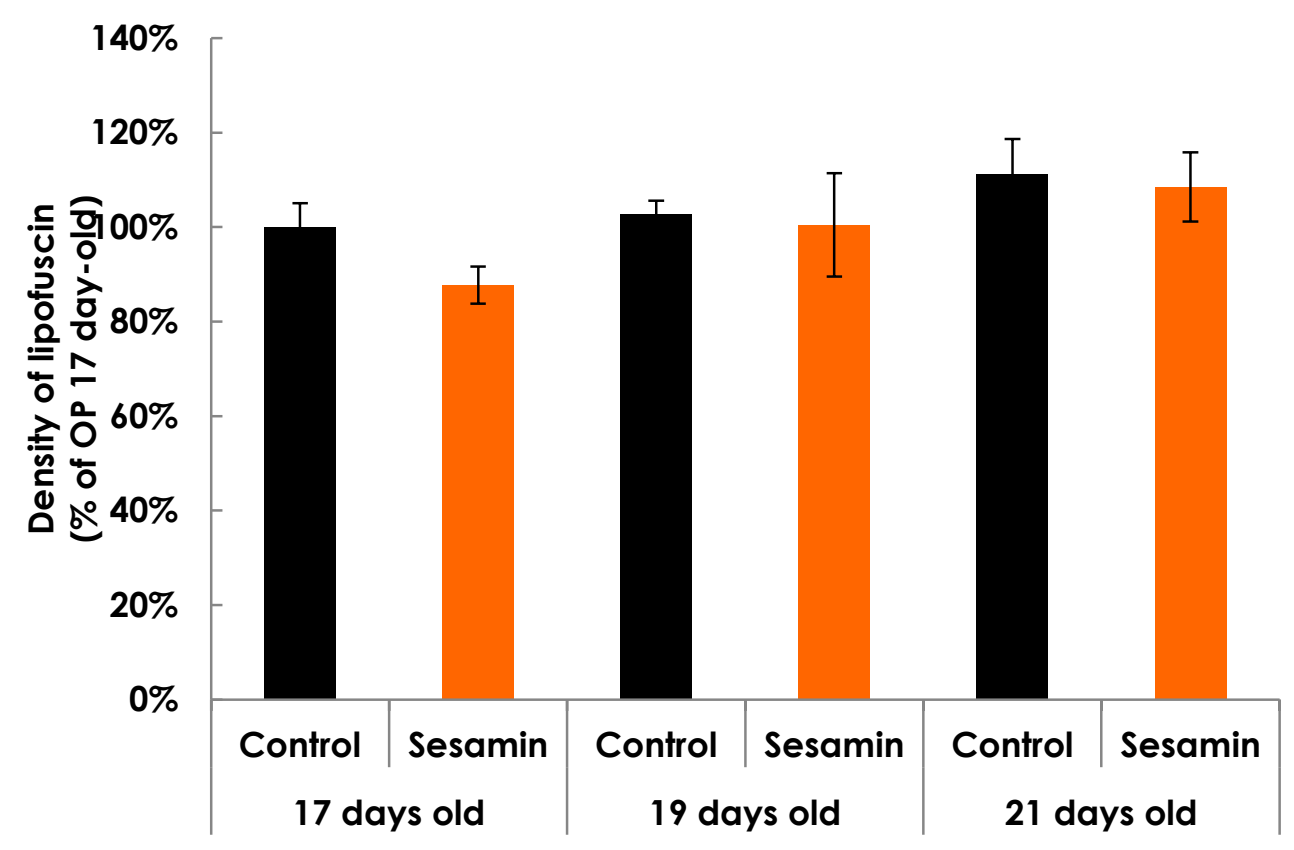

Online Resource Fig. 1 


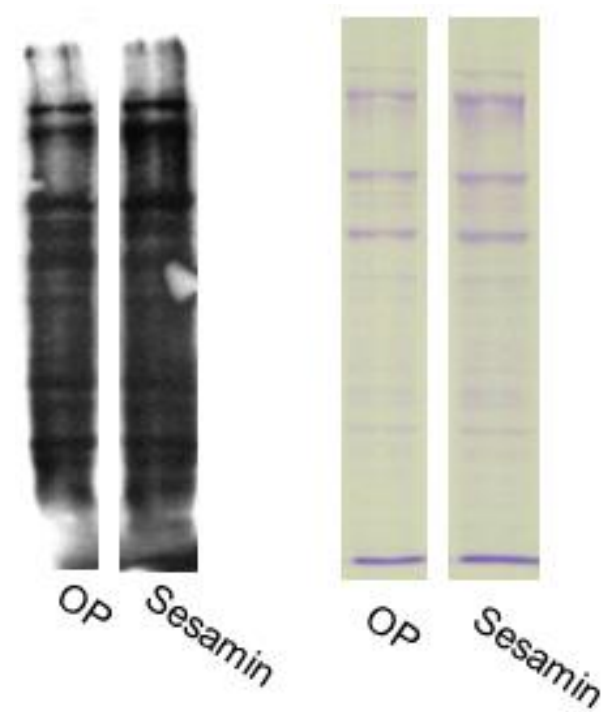

Online Resource Fig. 2a 


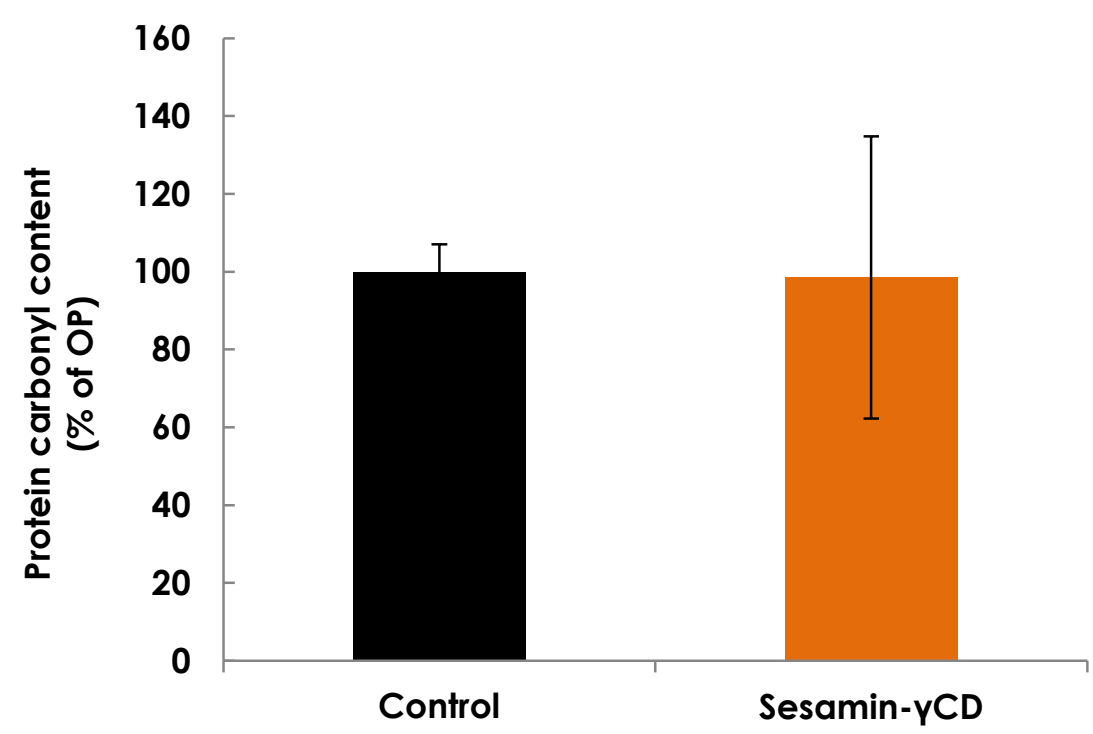

Online Resource Fig. 2b 


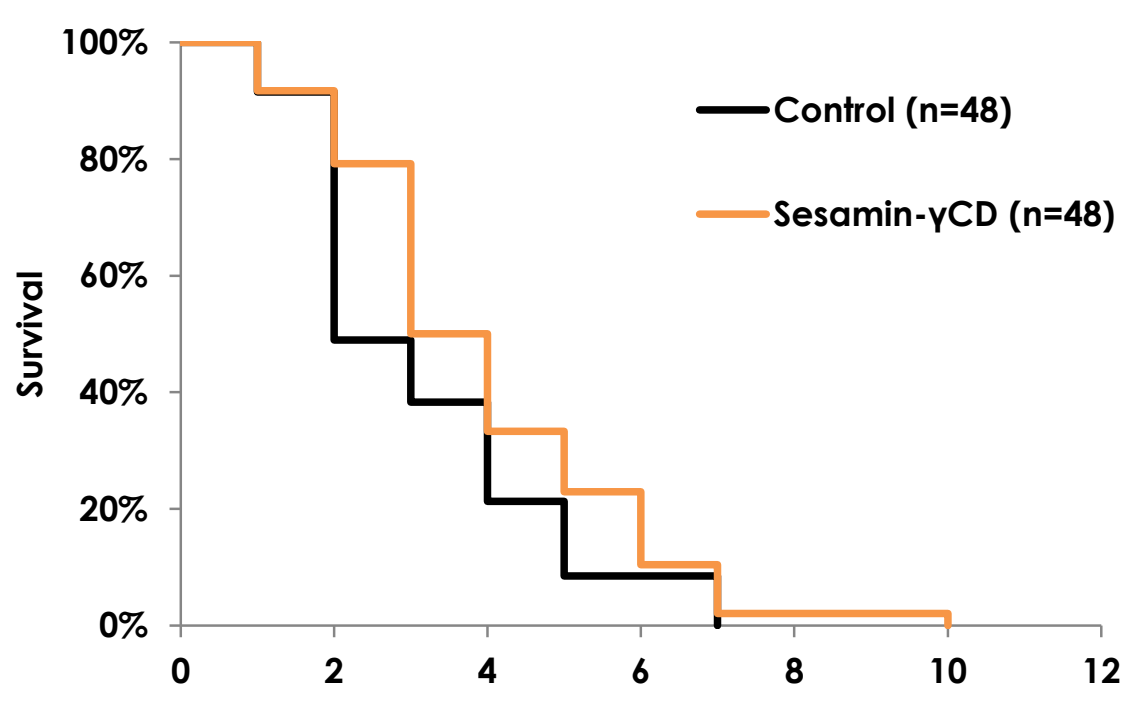

Hours in $\mathrm{CuCl}_{2}$ solution at 9 days old

Online Resource Fig. 3a 


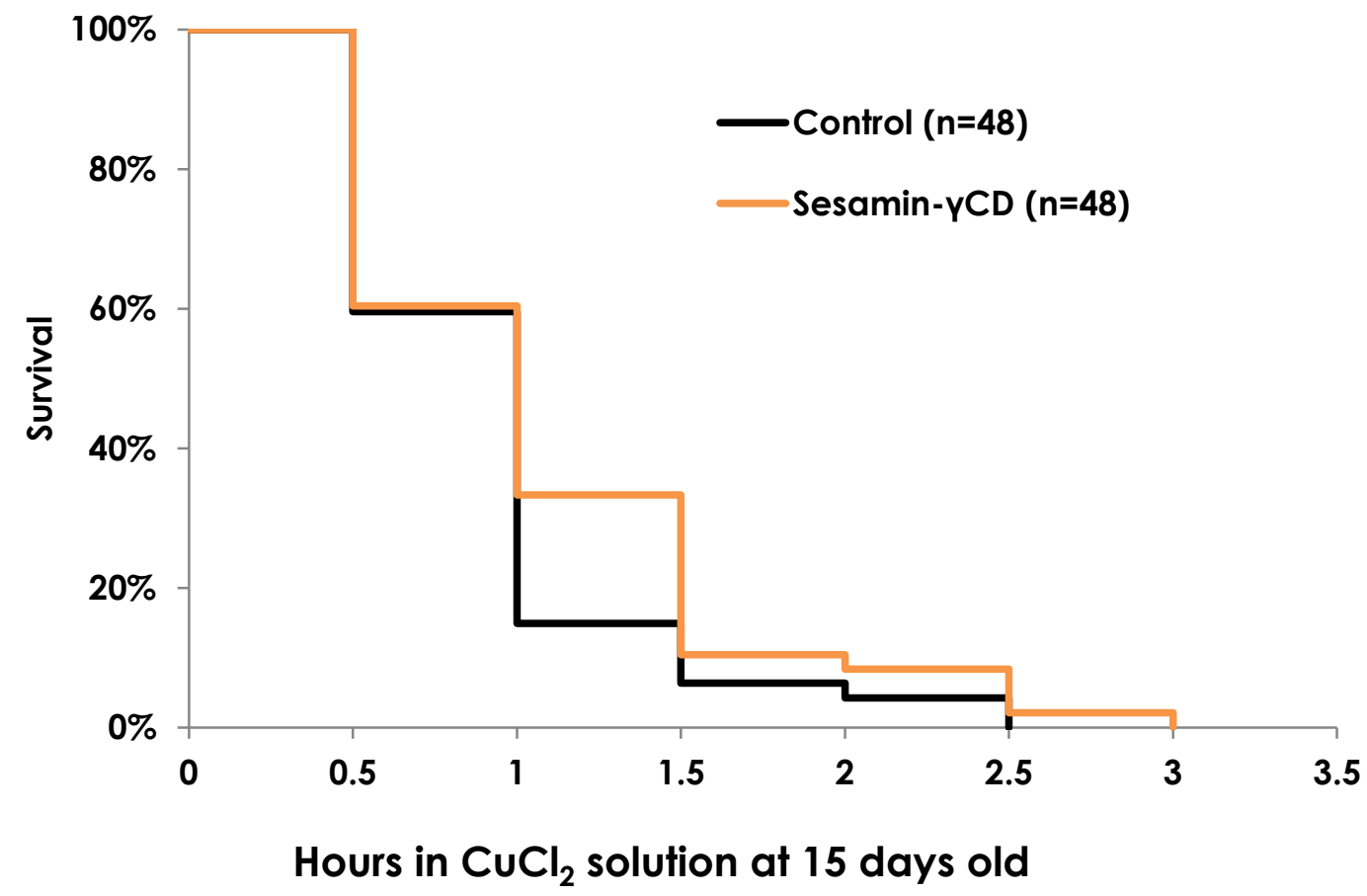

Online Resource Fig. 3b 


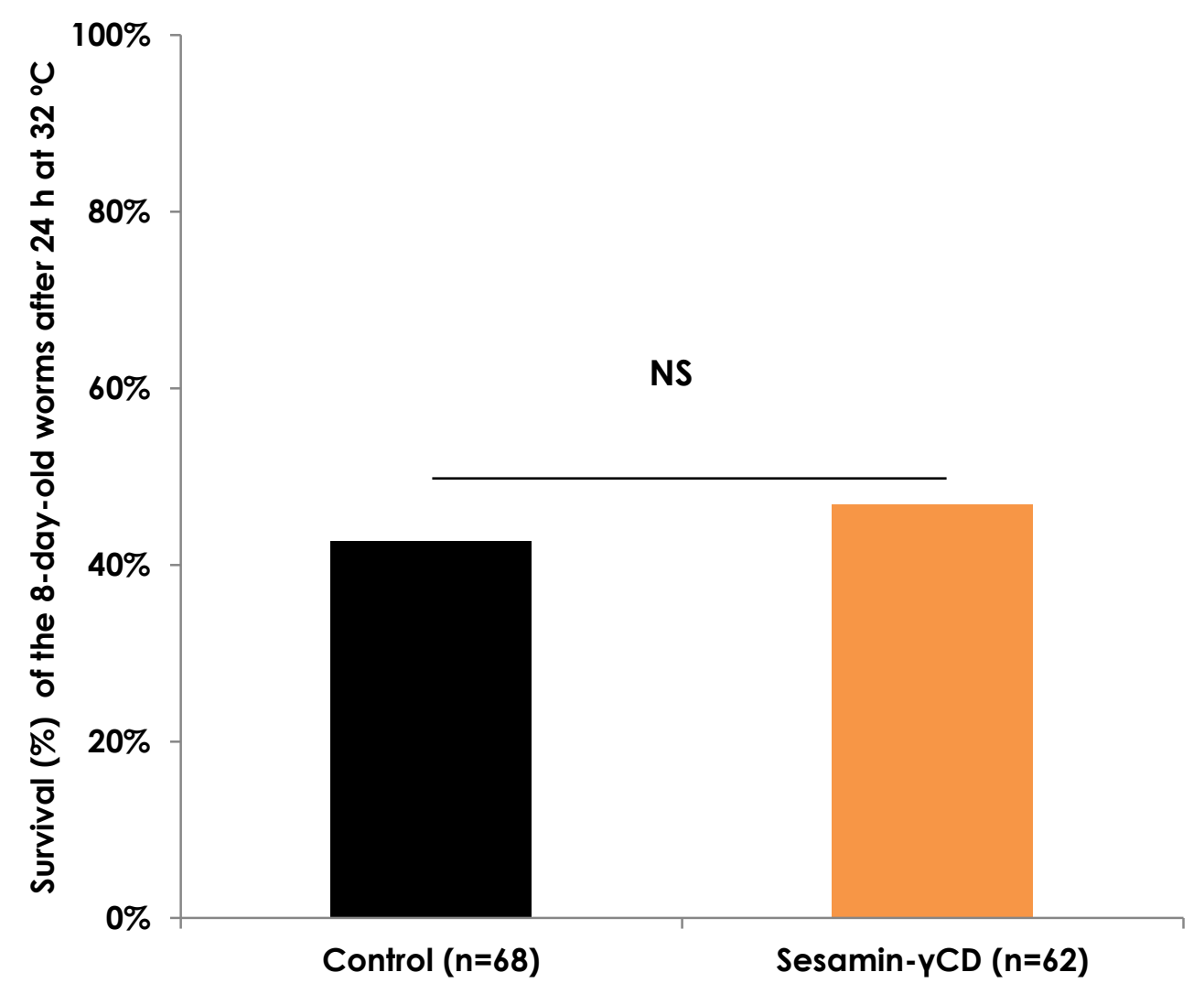

Online Resource Fig. 4a 


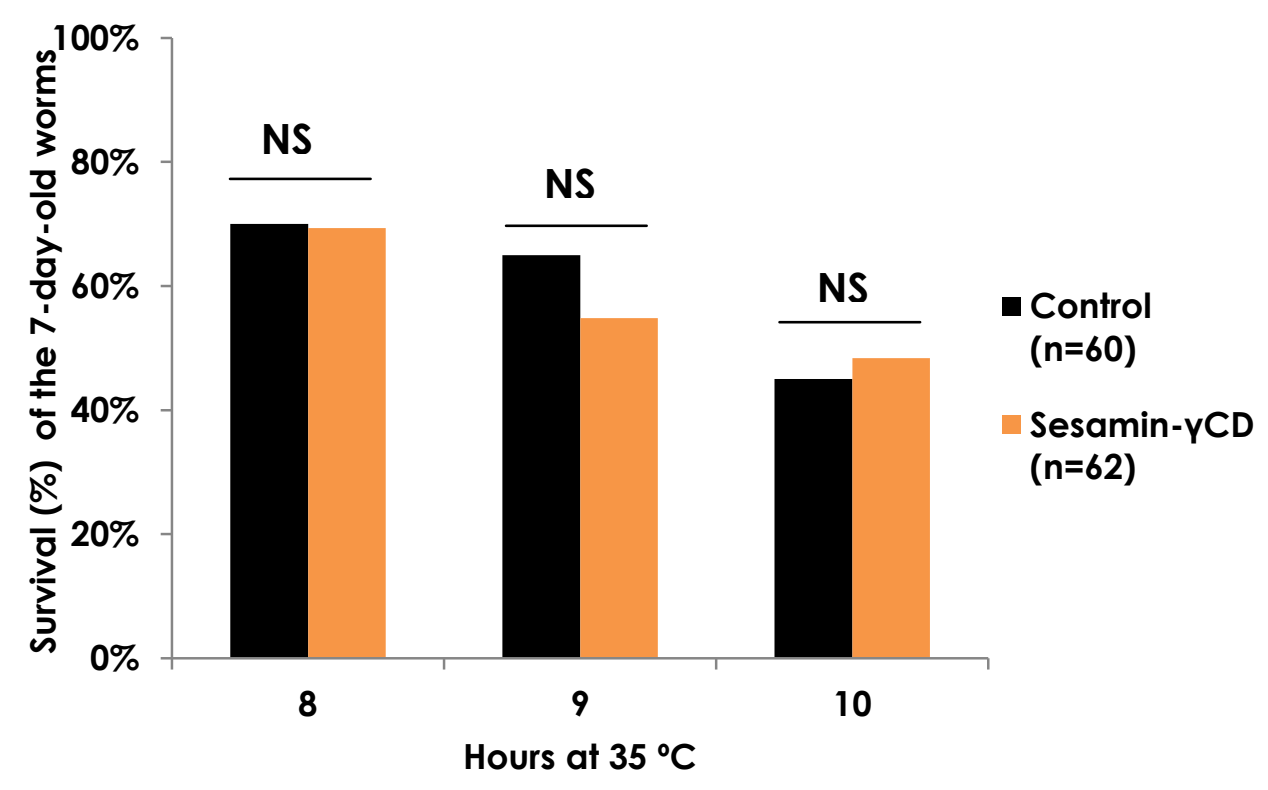

Online Resource Fig. 4b 


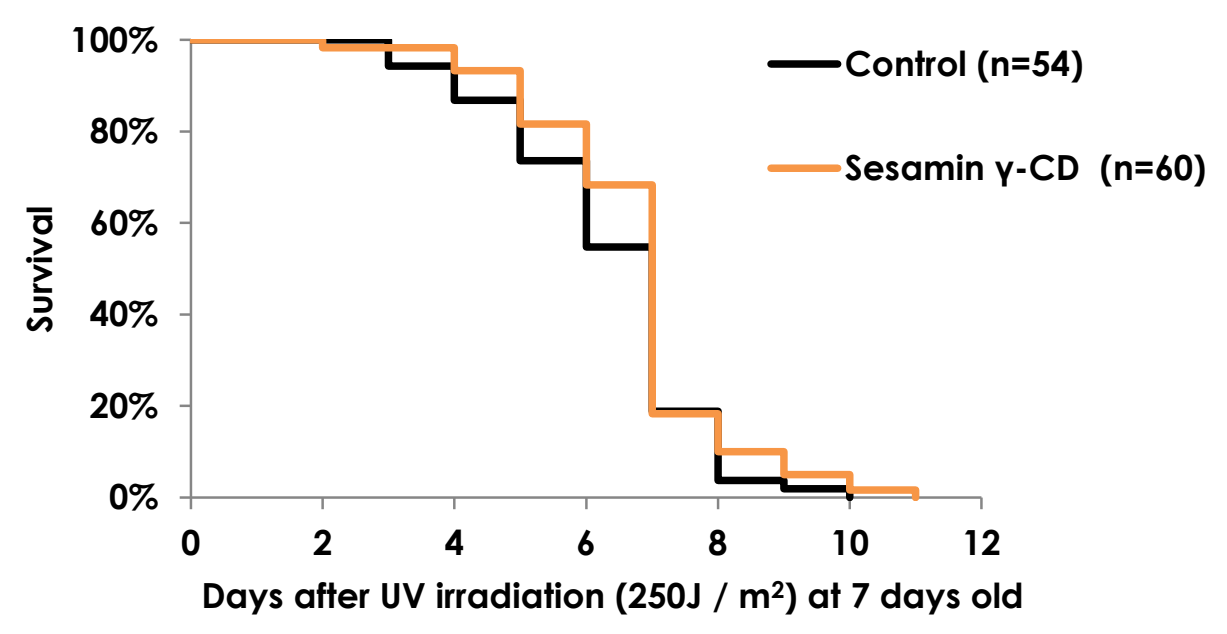

Online Resource Fig. 4c 


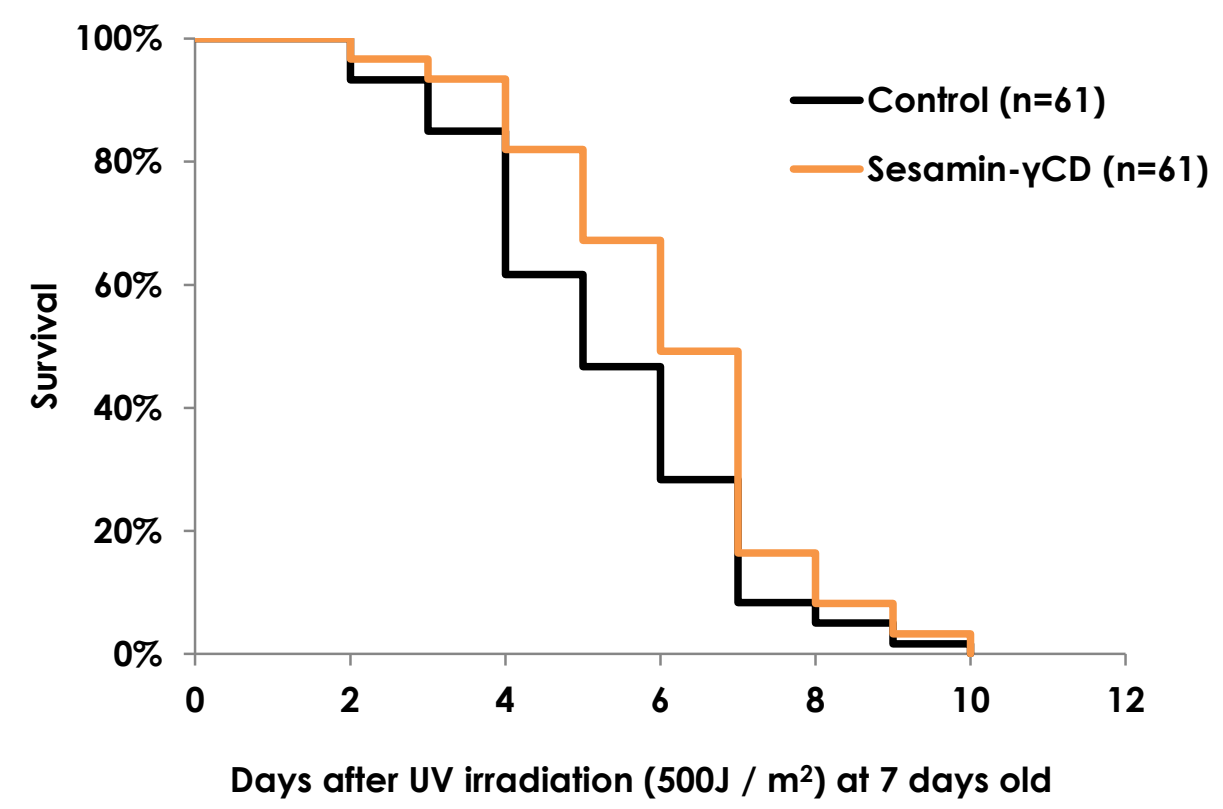

Online Resource Fig. 4d 


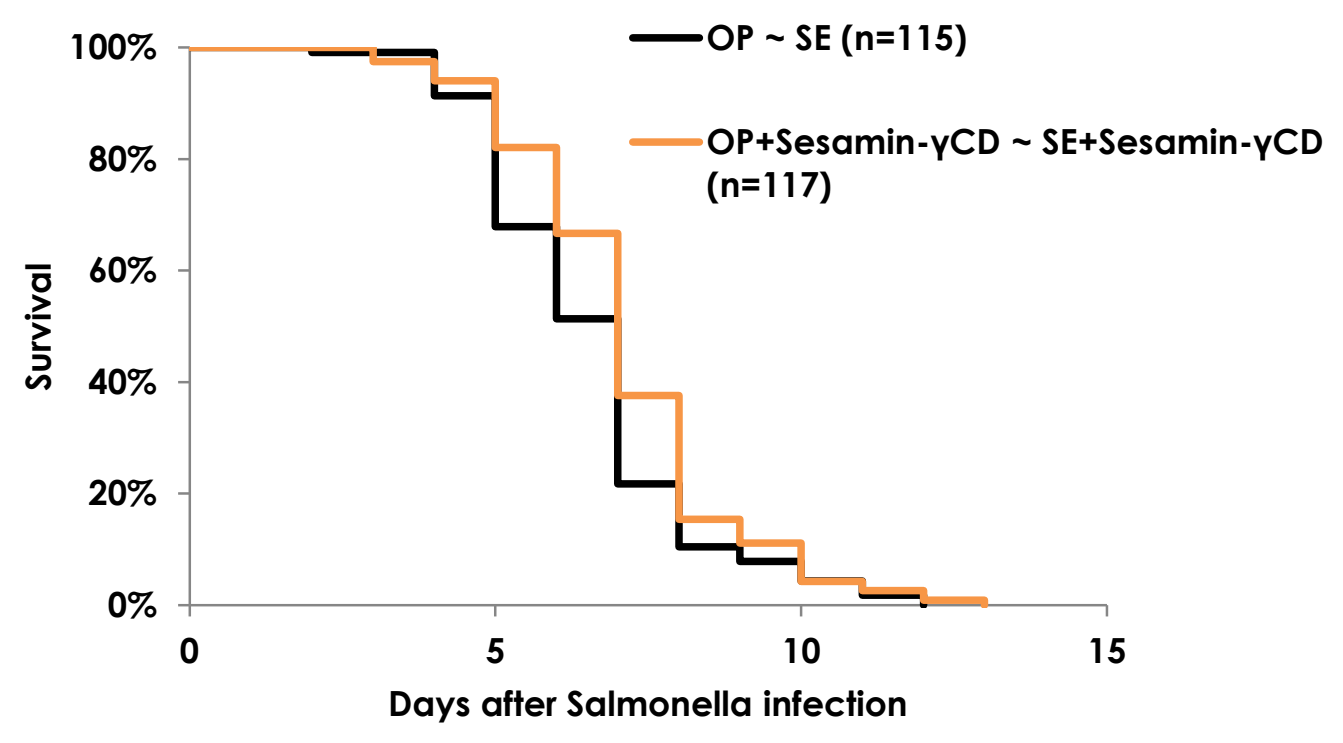

Online Resource Fig. 5 


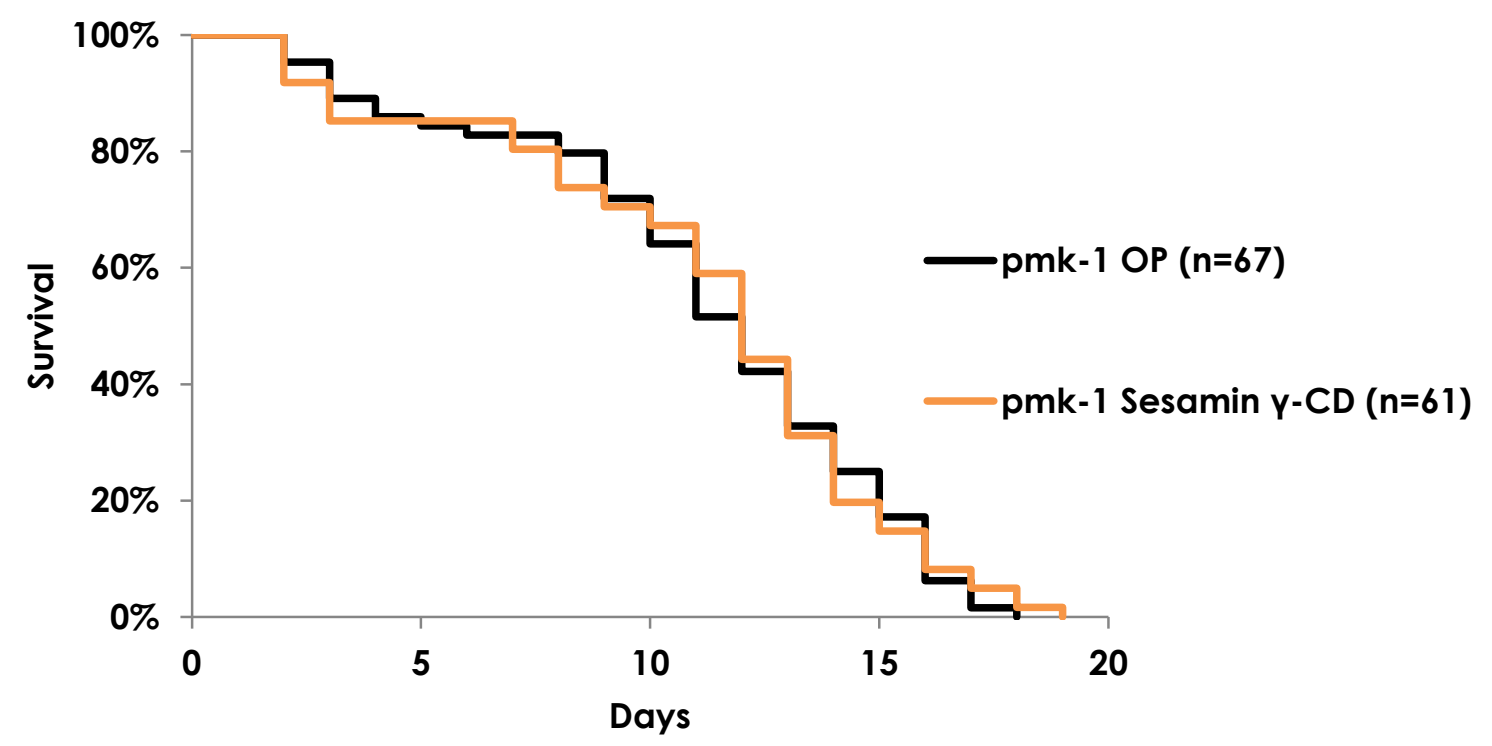

Online Resource Fig. 6a 


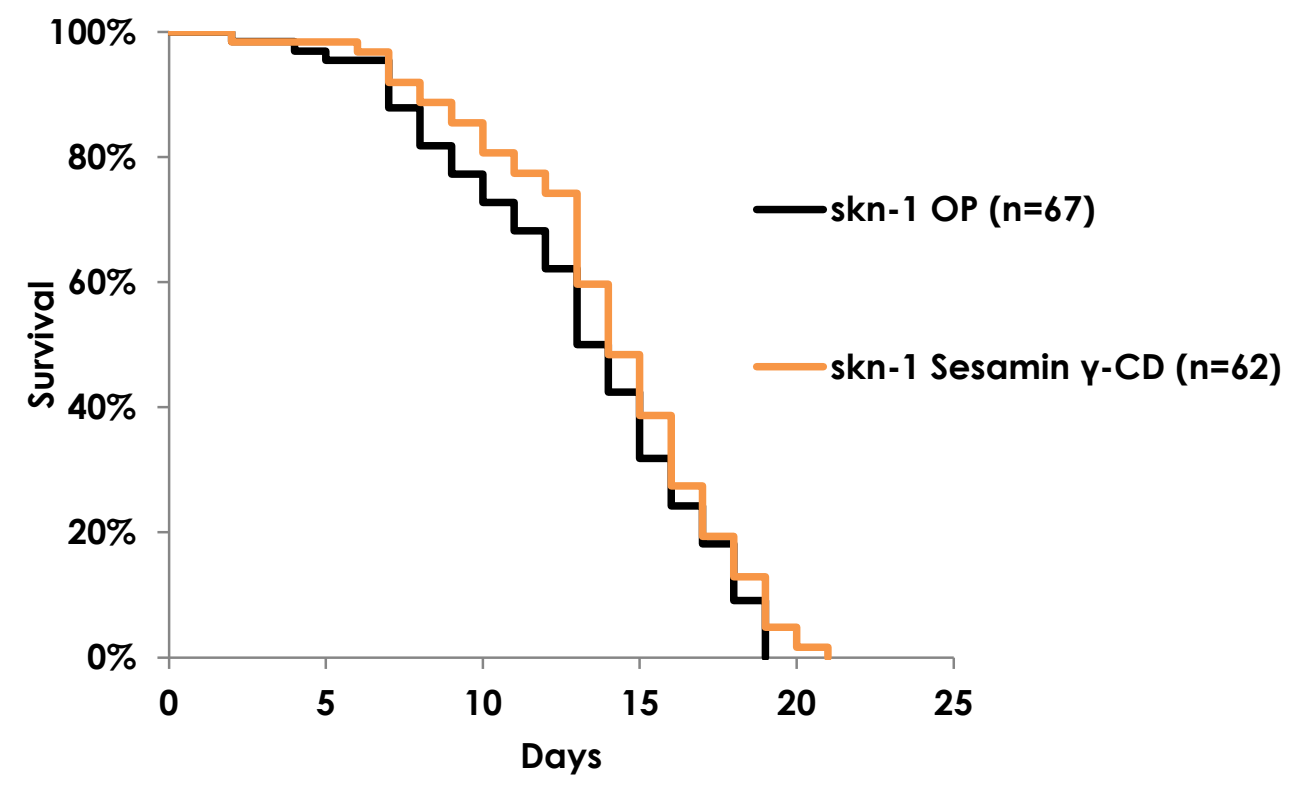

Online Resource Fig. 6b 


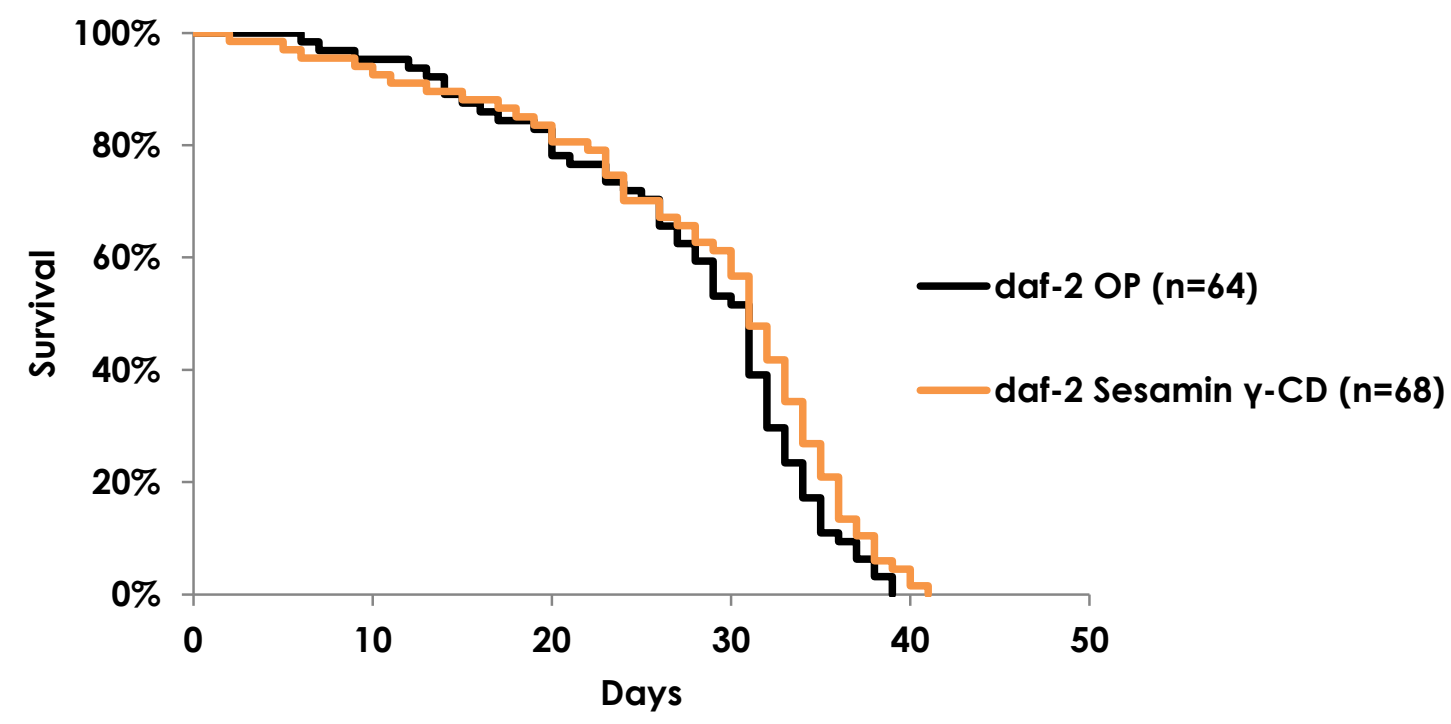

Online Resource Fig. 6c 


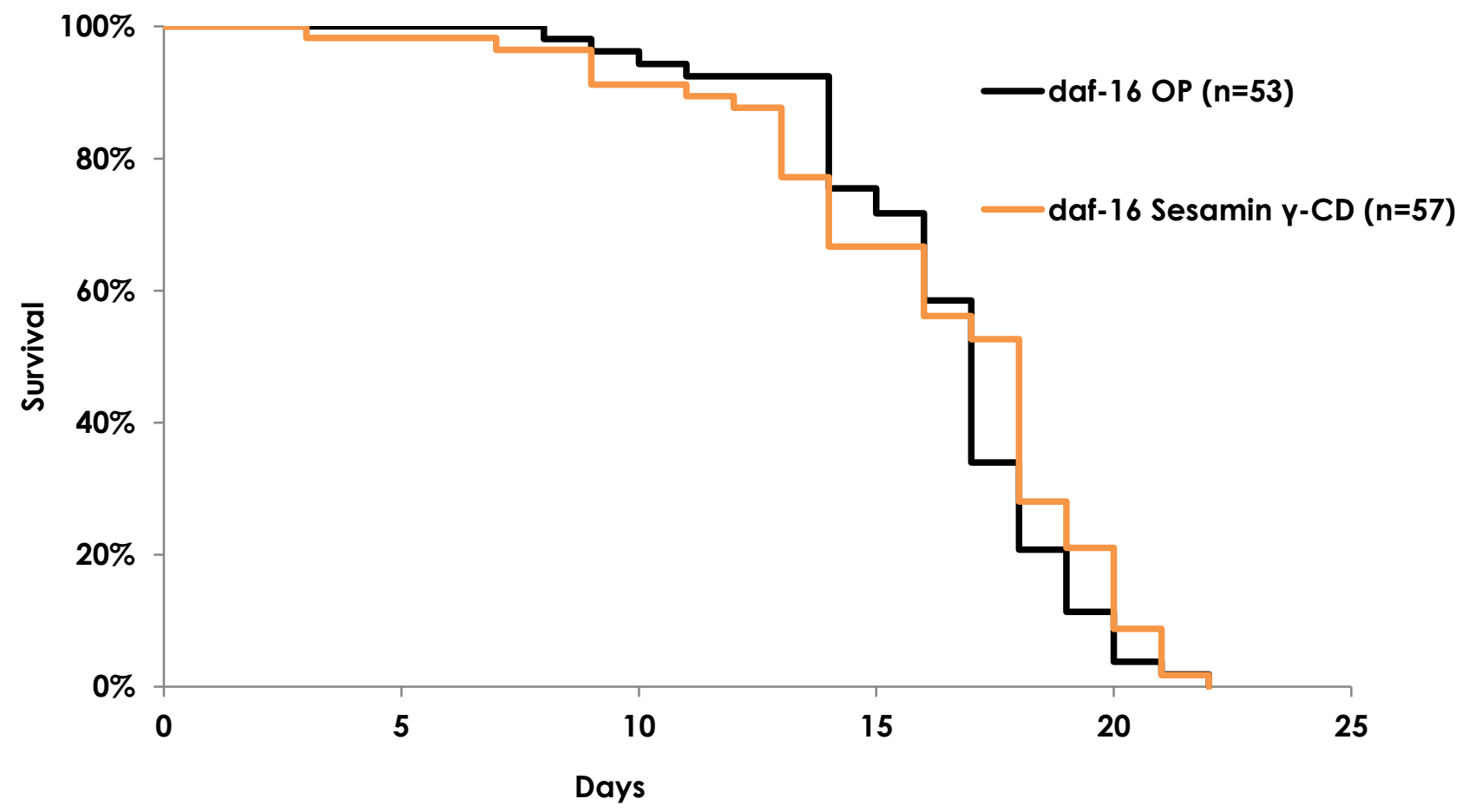

Online Resource Fig. 6d 


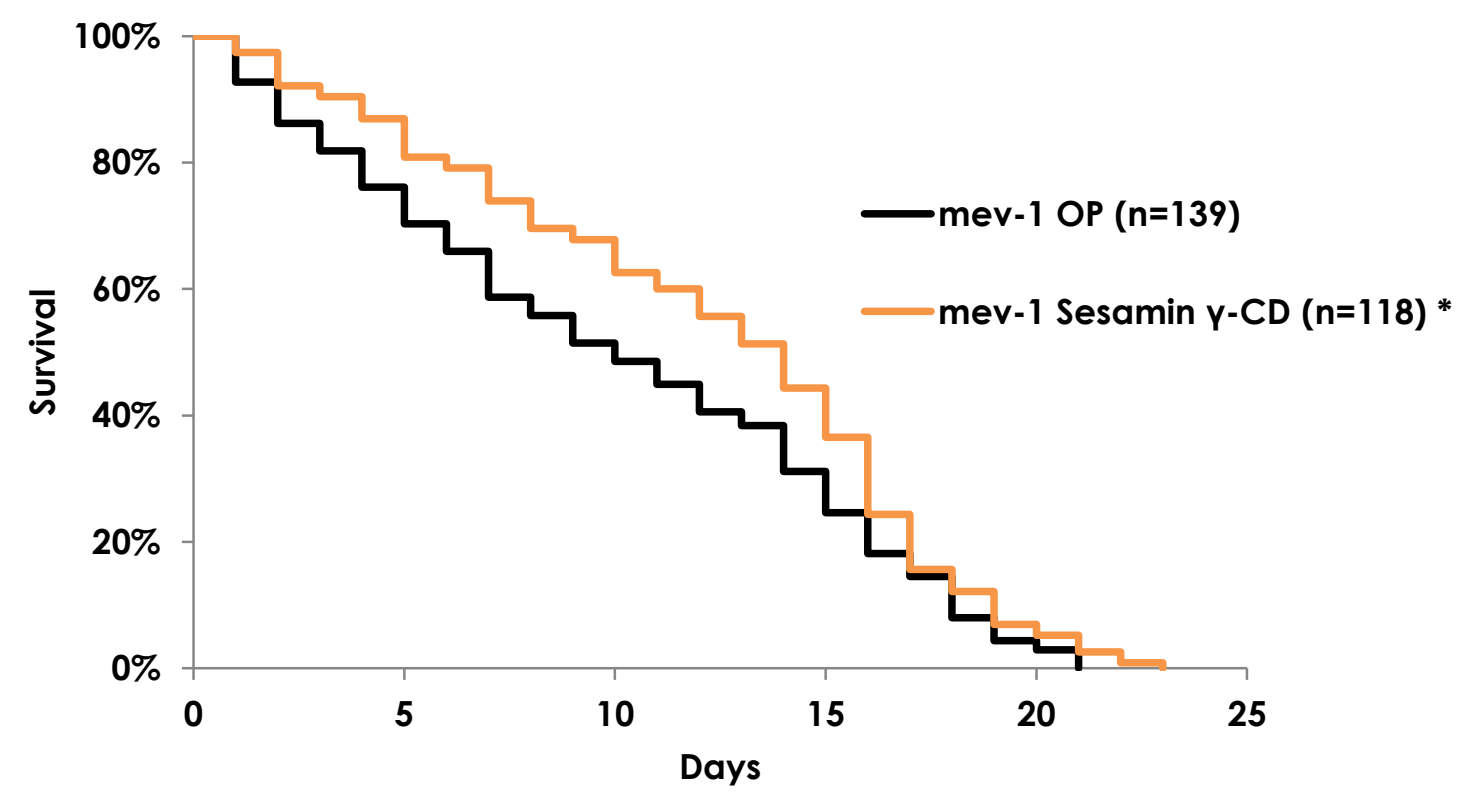

Online Resource Fig. 6e 